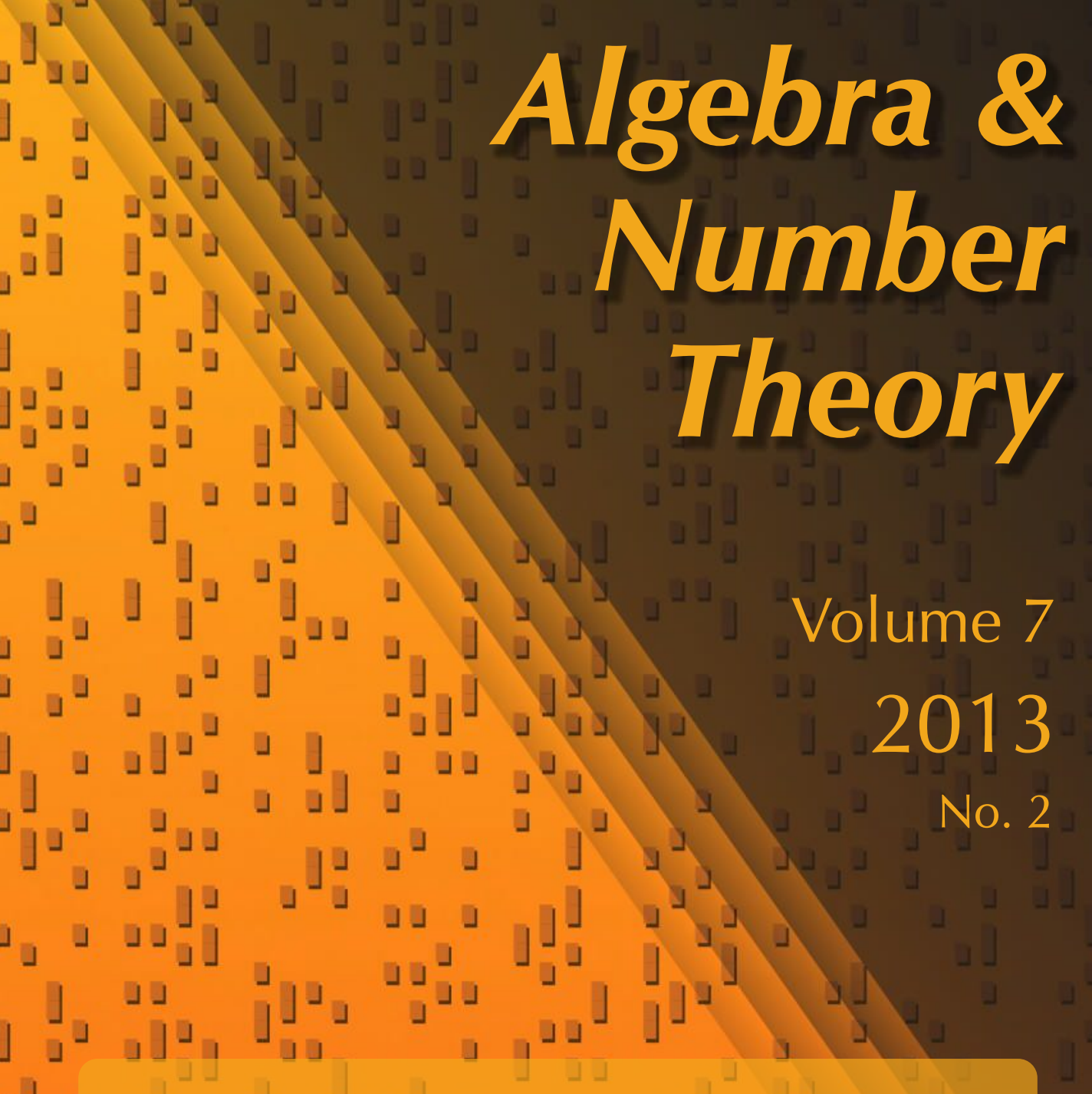

Arithmetic motivic Poincaré series of toric varieties

Helena Cobo Pablos and Pedro Daniel González Pérez

」

\lrcorner$\lrcorner$
\lrcorner 


\title{
Arithmetic motivic Poincaré series of toric varieties
}

\author{
Helena Cobo Pablos and Pedro Daniel González Pérez
}

\begin{abstract}
The arithmetic motivic Poincaré series of a variety $V$ defined over a field of characteristic zero is an invariant of singularities that was introduced by Denef and Loeser by analogy with the Serre-Oesterlé series in arithmetic geometry. They proved that this motivic series has a rational form that specializes to the Serre-Oesterlé series when $V$ is defined over the integers. This invariant, which is known explicitly for a few classes of singularities, remains quite mysterious. In this paper, we study this motivic series when $V$ is an affine toric variety. We obtain a formula for the rational form of this series in terms of the Newton polyhedra of the ideals of sums of combinations associated to the minimal system of generators of the semigroup of the toric variety. In particular, we explicitly deduce a finite set of candidate poles for this invariant.
\end{abstract}

\section{Introduction}

Let $S$ denote an irreducible and reduced algebraic variety defined over a field $k$ of characteristic zero. The set $H(S)$ of formal arcs of the form Spec $k \llbracket t \rrbracket \rightarrow S$ can be given the structure of scheme over $k$ (not necessarily of finite type). If $0 \in S$, we denote by $H(S)_{0}$ the subscheme of the arc space consisting of arcs in $H(S)$ with origin at 0 . The set $H_{m}(S)$ of $m$-jets of $S$ of the form Spec $k[t] /\left(t^{m+1}\right) \rightarrow S$ has the structure of algebraic variety over $k$. By a theorem of Greenberg, the image of the space of $\operatorname{arcs} H(S)$ by the natural morphism of schemes $j_{m}: H(S) \rightarrow H_{m}(S)$ that maps any arc to its $m$-jet is a constructible subset of $H_{m}(S)$.

It follows that $j_{m}(H(S))$ defines a class $\left[j_{m}(H(S))\right]$ in the Grothendieck ring of varieties $K_{0}\left(\operatorname{Var}_{k}\right)$ and also a class $\chi_{c}\left(\left[H_{m}(S)\right]\right) \in K_{0}\left(\mathrm{CHMot}_{k}\right)$ in the Grothendieck ring of Chow motives, where $\chi_{c}: K_{0}\left(\operatorname{Var}_{k}\right) \rightarrow K_{0}\left(\mathrm{CHMot}_{k}\right)$ is the unique ring homomorphism that maps the class of a smooth projective variety to its Chow motive (see [Gillet and Soulé 1996; Guillén and Navarro Aznar 2002]). We denote

Cobo Pablos is supported by FWO-Flanders project G031806N. González Pérez is supported by Programa Ramón y Cajal of the Ministerio de Ciencia e Innovación (MCI), Spain. Both authors are supported by MCI grant MTM2010-21740-C02-01.

MSC2010: primary 14M25; secondary 14J17, 14B05.

Keywords: arithmetic motivic Poincaré series, toric geometry, singularities, arc spaces. 
by $K_{0}^{\mathrm{mot}}\left(\operatorname{Var}_{k}\right)$ the image of $K_{0}\left(\operatorname{Var}_{k}\right)$ by the homomorphism $\chi_{c}$. We use the same symbol $\mathbf{L}$ to denote the class $\left[\mathbf{A}_{k}^{1}\right] \in K_{0}\left(\operatorname{Var}_{k}\right)$ and the class $\chi_{c}\left(\left[\mathbf{A}_{k}^{1}\right]\right) \in K_{0}^{\operatorname{mot}}\left(\operatorname{Var}_{k}\right)$.

Denef and Loeser [DL 2004] have defined various notions of motivic Poincaré series, motivated by some generating series in arithmetic geometry. Assume for simplicity that the variety $S$ is defined over the integers. We denote by $p$ a prime number and by $\mathbb{Z}_{p}$ the $p$-adic integers. For every positive integer $m$, the symbol $N_{p, m}(S)$ denotes the number of rational points of $S$ over $\mathbb{Z} / p^{m+1} \mathbb{Z}$ that can be lifted to rational points of $S$ over $\mathbb{Z}_{p}$ by the projection induced by the natural map $\mathbb{Z}_{p} \rightarrow \mathbb{Z} / p^{m+1} \mathbb{Z}$. The Serre-Oesterlé series of $S$ at the prime $p$ is

$$
P_{p}^{S}(T)=\sum_{m \geq 0} N_{p, m}(S) T^{m} \in \mathbb{Z} \llbracket T \rrbracket .
$$

The definition of the geometric motivic Poincaré series,

$$
P_{\text {geom }}^{S}(T)=\sum_{m \geq 0} \chi_{c}\left(\left[j_{m}(H)\right]\right) T^{m} \in K_{0}^{\operatorname{mot}}\left(\operatorname{Var}_{k}\right) \otimes \mathbb{Q} \llbracket T \rrbracket,
$$

is inspired by that of the Serre-Oesterle series. However, there is no specialization of the series $P_{\text {geom }}^{S}(T)$ into $P_{p}^{S}(T)$ in general [DL 2004].

Denef and Loeser studied the "motivic nature" of the series $P_{p}^{S}(T)$, passing through the Grothendieck ring $K_{0}\left(\right.$ Field $\left._{k}\right)$ of ring formulas over $k$. By Greenberg's theorem, for every $m$ there exists a formula $\psi_{m}$ over $k$, such that for any field extension $k \subset K$, the $m$-jets over $k$ that can be lifted to arcs defined over $K$ correspond to the tuples satisfying $\psi_{m}$ in $K$. It follows that $\psi_{m}$ defines an element $\left[\psi_{m}\right] \in K_{0}\left(\right.$ Field $\left._{k}\right)$. Then, Denef and Loeser defined a ring homomorphism $\chi_{f}$ : $K_{0}\left(\right.$ Field $\left._{k}\right) \rightarrow K_{0}^{\text {mot }}\left(\operatorname{Var}_{k}\right) \otimes \mathbb{Q}$. This homomorphism can be seen as a generalization of $\chi_{c}$, since the image by $\chi_{f}$ of the class of the ring formula defining a variety $V$ coincides with the class $\chi_{c}([V])$ in $K_{0}^{\operatorname{mot}}\left(\operatorname{Var}_{k}\right) \otimes \mathbb{Q}$. The arithmetic motivic Poincaré series of $S$ is defined as

$$
P_{\mathrm{ar}}^{S}(T)=\sum_{m \geq 0} \chi_{f}\left(\left[\psi_{m}\right]\right) T^{m} \in K_{0}^{\mathrm{mot}}\left(\operatorname{Var}_{k}\right) \otimes \mathbb{Q} \llbracket T \rrbracket .
$$

Denef [1984] proved the rationality of the series $P_{p}^{S}(T)$ using quantifier elimination results. Denef and Loeser [1999; 2001] proved the rationality of the series $P_{\text {geom }}^{S}(T)$ and $P_{\mathrm{ar}}^{S}(T)$ by using quantifier eliminations theorems, various forms of motivic integration, and the existence of resolution of singularities.

If $V$ is a variety defined over the integers and $p$ is a prime number, the symbol $N_{p}(V)$ denotes the number of rational points of $V$ over the field of $p$ elements. Denef and Loeser proved that the result of applying the operator $N_{p}$ to the motivic arithmetic series $P_{\mathrm{ar}}^{S}(T)$ provides the Serre-Oesterlé series $P_{p}^{S}(T)$ for almost all primes $p$. 
If we fix the origin of the arcs at a fixed point $0 \in S$, we obtain local versions of the series $P_{\mathrm{ar}}^{(S, 0)}(T)$ and $P_{\text {geom }}^{(S, 0)}(T)$, which are also rational; see [DL 1999; 2001]. The rationality proofs therein are qualitative in nature; in particular, there is no conjecture on the significance of the terms appearing in the denominator of the rational form of the series $P_{\mathrm{ar}}^{(S, 0)}(T)$ or in $P_{\mathrm{geom}}^{(S, 0)}(T)$.

The rational form of the series $P_{\mathrm{ar}}^{(S, 0)}(T)$ is known explicitly for a few classes of singularities. If $(S, 0)$ is an analytically irreducible germ of plane curve, the information provided by the series $P_{\mathrm{ar}}^{(S, 0)}(T)$ is equivalent to the data of the Puiseux pairs [DL 2001]. Nicaise [2005a] proved the equality of the geometric and arithmetic motivic Poincaré series in the case of varieties that admit a very special resolution of singularities, in particular for normal toric surfaces; see also [Nicaise 2005b; Lejeune-Jalabert and Reguera 2003]. He gave a criterion for the equality $P_{\mathrm{ar}}^{(S, 0)}(T)=$ $P_{\mathrm{geom}}^{(S, 0)}(T)$ for various classes of singularities and also an example of a normal toric threefold $\left(S_{0}, 0\right)$ such that the series $P_{\mathrm{ar}}^{\left(S_{0}, 0\right)}(T)$ and $P_{\text {geom }}^{\left(S_{0}, 0\right)}(T)$ are different. Some features of the motivic arithmetic series are studied for quasi-ordinary singularities in [Rond 2009].

In this paper, we describe the arithmetic motivic Poincare series of an affine toric variety $Z^{\Lambda}=\operatorname{Spec} k[\Lambda]$, in terms of the semigroup $\Lambda$. We assume that $\Lambda$ is a semigroup of finite type of a rank $d$ lattice $M$ (lattice of characters), which generates $M$ as a group, and such that the cone $\mathbb{R}_{\geq 0} \Lambda$ contains no lines. In this situation, there is a unique minimal system of generators $e_{1}, \ldots, e_{n}$ of the semigroup $\Lambda$. The monomial ideal $\left(X^{e_{i}}\right)_{i=1, \ldots, n} \subset k[\Lambda]$ is maximal, and defines the distinguished point $0 \in Z^{\Lambda}$. In this paper we consider other monomial ideals as the logarithmic jacobian ideals $\mathscr{F}_{l}$, generated by monomials of the form $X^{u}$ for $u$ in the set

$$
\left\{e_{i_{1}}+\cdots+e_{i_{l}} \mid e_{i_{1}} \wedge \cdots \wedge e_{i_{l}} \neq 0\right\}
$$

for $l=1, \ldots, d$ (see [Cobo Pablos and González Pérez 2012]), and the ideals of sums of combinations $\mathscr{C}_{j}$, defined by monomials $X^{w}$, with $w$ in the set

$$
\left\{e_{i_{1}}+\cdots+e_{i_{j}} \mid\left\{i_{1}, \ldots, i_{j}\right\} \in\left(\begin{array}{c}
\{1, \ldots, n\} \\
j
\end{array}\right)\right\},
$$

where $\left(\begin{array}{c}\{1, \ldots, n\} \\ j\end{array}\right)$ denotes the set of combinations of $j$ elements of $\{1, \ldots, n\}$ for $j=1, \ldots, n$.

We study the motivic arithmetic series $P_{\mathrm{ar}}^{\left(Z^{\Lambda}, 0\right)}(T)$ by extending the approach we used in [CoGP 2010; 2012] to describe the geometric motivic Poincare series of toric and quasi-ordinary singularities.

For convenience, we explain the methods and results first when the variety $Z^{\Lambda}$ is normal. The set $j_{m}\left(H\left(Z^{\Lambda}\right)_{0}\right)$ of $m$-jets of arcs through $\left(Z^{\Lambda}, 0\right)$ is constructible; it is a finite disjoint union of locally closed subsets of the form $j_{m}\left(H_{v}^{*}\right)$ [Cobo Pablos and González Pérez 2012]. Here $H_{v}^{*}$ denotes the set of $\operatorname{arcs}$ through $\left(Z^{\Lambda}, 0\right)$ that 
have generic point in the torus and a given order $v \in M^{*}$. The set $H_{v}^{*}$ is an orbit of the natural action of the arc space of the torus on the arc space of the toric variety $Z^{\Lambda}$ [Ishii 2004; 2005].

We describe the class, denoted by $\chi_{f}\left(\left[j_{m}\left(H_{v}^{*}\right)\right]_{f}\right)$, of the formula defining the locally closed subset $j_{m}\left(H_{v}^{*}\right)$ in terms of the Newton polyhedra of the logarithmic jacobian ideals and the degree of a certain Galois cover. This Galois cover reflects the relation between the initial coefficients of the arcs in $H_{v}^{*}$ and the initial coefficients of the $m$-jets in $j_{m}\left(H_{v}^{*}\right)$; see Section 5 .

A key point in the description of the rational form of the series $P_{\mathrm{ar}}^{\left(Z^{\Lambda}, 0\right)}(T)$ is that using the ideals $\mathscr{C}_{j}$, we can refine a finite partition of the set of possible pairs $\{(\nu, m)\}$, which was defined in [Cobo Pablos and González Pérez 2012] to describe the sum of $P_{\mathrm{geom}}^{\left(Z^{\Lambda}, 0\right)}(T)$. If $(v, m)$ and $\left(v^{\prime}, m^{\prime}\right)$ belong to the same subset of this refinement, then the degrees of the Galois covers associated to $j_{m}\left(H_{v}^{*}\right)$ and $j_{m^{\prime}}\left(H_{v^{\prime}}^{*}\right)$ coincide (see Sections 6 and 7). Using these partitions, we decompose the series $P_{\mathrm{ar}}^{\left(Z^{\Lambda}, 0\right)}(T)$ as a sum of a finite number of contributions. The main result is a formula for the rational form of $P_{\mathrm{ar}}^{\left(Z^{\Lambda}, 0\right)}(T)$ (see Theorem 11.4 and Corollary 10.4). The proofs pass by the results on the generating function of the projection of the set of integral points in the interior of a rational polyhedral cone; see [Cobo Pablos and González Pérez 2012]. The denominator of $P_{\mathrm{ar}}^{\left(Z^{\Lambda}, 0\right)}(T)$ is a finite product of terms of the form $1-\mathbf{L}^{a} T^{b}$ with $a \geq 0$ and $b>0$, which are determined explicitly in terms of the ideals of sums of combinations $\mathscr{C}_{j}$. The integers $a$ and $b$ can be described in terms of the orders of vanishing of the ideals $\mathscr{C}_{j}$ and $\mathscr{F}_{l}$ at the codimension-one orbits of various toric modifications given by the Newton polyhedra of the ideals $\mathscr{C}_{j}$ (see Remark 10.8). In the normal toric case, we obtain a formula for $P_{\mathrm{ar}}^{Z^{\Lambda}}(T)$ in terms of arithmetic motivic series at the distinguished points of the orbits.

In the nonnormal case, we obtain in a similar way a formula for the rational form of $P_{\mathrm{ar}}^{\left(Z^{\Lambda}, 0\right)}(T)$ and the factors of its denominator. The main difference is that we have to consider contributions of jets of arcs with generic point in the various orbits of $Z^{\Gamma}$. We deduce a formula for the difference $P_{\mathrm{geom}}^{\left(Z^{\Lambda}, 0\right)}(T)-P_{\mathrm{ar}}^{\left(Z^{\Lambda}, 0\right)}(T)$ and we give a criterion for the equality of these two series that generalizes the one given by Nicaise [2005b] (see Proposition 10.5 and Corollary 10.6).

The paper is organized as follows. In Sections 1 and 2 we introduce the Grothendieck rings, the arc and jet spaces, and the motivic Poincaré series. The notations on toric varieties, their monomial ideals, and their arcs are introduced in Sections 3 and 4. The computation of the class $\chi_{f}\left(\left[j_{m}\left(H_{v}^{*}\right)\right]_{f}\right)$ is given in Section 5. Sections 6 and 7 deal with the partitions associated to sequences of monomial ideals. The main results are stated and proved in Sections 8, 9 and 10. In the case of normal toric varieties, some features of the computation can be simplified (see Section 11). We discuss some examples in Section 12. 


\section{Grothendieck rings of varieties and of ring formulas}

The Grothendieck ring $K_{0}\left(\operatorname{Var}_{k}\right)$ of $k$-varieties is the free abelian group of isomorphism classes $[X]$ of $k$-varieties $X$ modulo the relations $[X]=\left[X^{\prime}\right]+\left[X \backslash X^{\prime}\right]$ if $X^{\prime}$ is closed in $X$, and where the product is defined by $[X]\left[X^{\prime}\right]=\left[X \times X^{\prime}\right]$. We denote by $\mathbf{L}:=\left[\mathbf{A}_{k}^{1}\right]$ the class of the affine line. If $C$ is a constructible subset of some variety $X$, that is, a disjoint union of finitely many locally closed subvarieties $A_{i}$ of $X$, then $[C] \in K_{0}\left(\operatorname{Var}_{k}\right)$ is well-defined as $[C]:=\sum_{i}\left[A_{i}\right]$ independently of the representation. Bittner [2004] proved, using the weak factorization theorem, that the ring $K_{0}\left(\operatorname{Var}_{k}\right)$ is generated by classes of smooth projective $k$-varieties, modulo relations of the form $[W]-[E]=[X]-[Y]$, where $Y \subset X$ is a closed subvariety, and $W$ is the blowing up of $X$ along $Y$ with exceptional divisor $E$.

We refer to [Scholl 1994; Gillet and Soulé 1996; Guillén and Navarro Aznar 2002] for the definition of the category of Chow motives. Roughly speaking, its definition involves replacing the category of smooth projective algebraic varieties over $k$ by a category with basically the same objects, and whose morphisms are suitable correspondences modulo rational equivalence. There exists a unique ring homomorphism

$$
\chi_{c}: K_{0}\left(\operatorname{Var}_{k}\right) \rightarrow K_{0}\left(\mathrm{CHMot}_{k}\right)
$$

that maps the class of a smooth projective variety over $k$ to its Chow motive, where $K_{0}\left(\mathrm{CHMot}_{k}\right)$ denotes the Grothendieck ring of the category of Chow motives over $k$ (with coefficients in $\mathbb{Q}$ ). This fundamental theorem, which is due to Gillet and Soulé [1996] and Guillén and Navarro Aznar [2002], can be seen also in terms of Bittner's result. We refer to [Gillet and Soulé 1996; Guillén and Navarro Aznar 2002; Bittner 2004] for precise definitions and proofs and to [Scholl 1994] for a survey on the notion of motives. We denote by $K_{0}^{\mathrm{mot}}\left(\operatorname{Var}_{k}\right)$ the image of $K_{0}\left(\operatorname{Var}_{k}\right)$ in $K_{0}\left(\mathrm{CHMot}_{k}\right)$ under this homomorphism.

Notice that the image of $\mathbf{L}$ in $K_{0}^{\mathrm{mot}}\left(\operatorname{Var}_{k}\right)$, which we denote with the same symbol, is not a zero divisor in $K_{0}^{\operatorname{mot}}\left(\operatorname{Var}_{k}\right)$ since it is a unit in $K_{0}\left(\mathrm{CHMot}_{k}\right)$. However, it seems that it is not known if $\mathbf{L}$ is a zero divisor in $K_{0}\left(\operatorname{Var}_{k}\right)$.

A ring formula $\psi$ over $k$ is a first-order formula in the language of $k$-algebras and free variables $x_{1}, \ldots, x_{n}$, that is, the formula $\psi$ is built from boolean combinations ("and", "or", "not") of polynomial equations over $k$ and existential and universal quantifiers. The Grothendieck ring $K_{0}\left(\right.$ Field $\left._{k}\right)$ of ring formulas over $k$ is generated by symbols $[\psi]$, where $\psi$ is a ring formula over $k$, subject to the relations

$$
\left[\psi_{1} \vee \psi_{2}\right]=\left[\psi_{1}\right]+\left[\psi_{2}\right]-\left[\psi_{1} \wedge \psi_{2}\right]
$$

if $\psi_{1}$ and $\psi_{2}$ have the same free variables, and $\left[\psi_{1}\right]=\left[\psi_{2}\right]$ if there exists a ring formula $\Psi$ over $k$ such that when interpreted in any field, $K \supseteq k$ provides the graph of a bijection between the tuples of elements of $K$ satisfying $\psi_{1}$ and those satisfying 
$\psi_{2}$. The ring multiplication is induced by the conjunction of formulas in disjoint sets of variables [DL 2001]. Denef and Loeser defined a ring homomorphism

$$
\chi_{f}: K_{0}\left(\text { Field }_{k}\right) \rightarrow K_{0}^{\operatorname{mot}}\left(\operatorname{Var}_{k}\right) \otimes \mathbb{Q} .
$$

They proved that this homomorphism is characterized by two conditions. The first one is that for any ring formula $\psi$ that is a conjunction of polynomial equations over $k$, the element $\chi_{f}([\psi])$ is equal to the class $\chi_{c}([V])$ in $K_{0}^{\text {mot }}\left(\operatorname{Var}_{k}\right) \otimes \mathbb{Q}$ of the variety $V$ defined by $\psi$. The second condition, which is more technical, expresses that certain relations should hold in terms of unramified Galois coverings over $k$. We refer to [DL 2001; 2004] for the precise statement. In the simplest case it implies the following:

Example 1.1 [DL 2004, Example 6.4.3]. If $n \geq 1$ is a fixed integer, $k$ is a field containing all $n$-th roots of unity, and $\psi$ is the ring formula

$$
\psi: \text { there exists y such that } x=y^{n} \text { and } x \neq 0
$$

then we have that $\chi_{f}([\psi])=(1 / n)(\mathbf{L}-1)$.

Lemma 1.2. Let $\psi$ be the ring formula whose interpretation in any field $K \supseteq k$ provides the set of $K$-rational points of $T$ that lift to $K$-rational points of $T^{\prime}$ by $a$ Galois covering $T^{\prime} \rightarrow T$ of degree $n$ of $d$-dimensional algebraic $k$-tori. If the field $k$ contains all the $n$-th roots of unity, then we have that $\chi_{f}([\psi])=(1 / n)(\mathbf{L}-1)^{d}$.

Proof. The morphism $T^{\prime} \rightarrow T$ induces a finite index inclusion of the corresponding character group $M \subseteq M^{\prime}$, and hence a map of $k$-algebras $k[M] \hookrightarrow k\left[M^{\prime}\right]$. By the classification theorem of finitely generated abelian groups applied to $M^{\prime} / M$, there exists a basis $\left\{v_{1}, \ldots, v_{d}\right\}$ of $M^{\prime}$ and unique integers $b_{1}\left|b_{2}\right| \cdots \mid b_{d}$, where $\mid$ denotes division, such that $\left\{b_{1} v_{1}, \ldots, b_{d} v_{d}\right\}$ is a basis of $M$ and $n=b_{1} \ldots b_{d}$. It follows that the map of coordinate rings $K[M] \hookrightarrow K\left[M^{\prime}\right]$ expresses in coordinates as $K\left[z_{1}^{ \pm b_{1}}, \ldots, z_{d}^{ \pm b_{d}}\right] \hookrightarrow K\left[z_{1}^{ \pm 1}, \ldots, z_{d}^{ \pm 1}\right]$. We deduce that the ring formula $\psi$ is the conjunction of formulas $\psi_{i}$ : there exists $y_{i}$ such that $x_{i}=y_{i}^{b_{i}}$ and $x_{i} \neq 0$ for $i=1, \ldots, d$, where the variables $x_{1}, \ldots, x_{d}$ are independent. Then we get that

$$
\chi_{f}([\psi])=\frac{1}{b_{1} \ldots b_{d}}(\mathbf{L}-1)^{d}
$$

Remark 1.3. Denef and Loeser defined the map $\chi_{f}$ by factoring it through the Grothendieck ring $K_{0}\left(\mathrm{PFF}_{k}\right)$ of ring formulas for the category of pseudofinite fields containing $k$. See [DL 2001; 2004; 2002]. 


\section{Arcs, jets and motivic Poincaré series}

We start this section by recalling the definition of the space of arcs of a variety $S$. We assume for simplicity that $S$ is an affine irreducible and reduced algebraic variety defined over a field $k$ of characteristic zero.

For any integer $m \geq 0$, the functor from the category of $k$-algebras to the category of sets, sending a $k$-algebra $R$ to the set of $R[t] /\left(t^{m+1}\right)$-rational points of $S$, is representable by a $k$-scheme $H_{m}(S)$ of finite type over $k$, called the $m$-jet scheme of $S$. The natural maps induced by truncation $j_{m}^{m+1}: H_{m+1}(S) \rightarrow H_{m}(S)$ are affine, and hence the projective limit $H(S):=\lim _{m} H_{m}(S)$ is a $k$-scheme, not necessarily of finite type, called the arc space of $S$.

In what follows, we consider the schemes $H_{m}(S)$ and $H(S)$ with their reduced structure. We have natural morphisms $j_{m}: H(S) \rightarrow H_{m}(S)$. By an arc we mean a $k$-rational point of $H(S)$, that is, a morphism Spec $k \llbracket t \rrbracket \rightarrow S$. By an $m$-jet we mean a $k$-rational point of $H_{m}(S)$, that is, a morphism Spec $k[t] /\left(t^{m+1}\right) \rightarrow S$. The origin of the arc (respectively of the $m$-jet) is the image of the closed point 0 of Spec $k \llbracket t \rrbracket$ (respectively of Spec $k[t] /\left(t^{m+1}\right)$ ).

If $Z \subset S$ is a closed subvariety, then $H(S)_{Z}:=j_{0}^{-1}(Z)$ (respectively $H_{m}(S)_{Z}:=$ $\left(j_{0}^{m}\right)^{-1}(Z)$ ) denotes the subscheme of $H(S)$ (respectively of $H_{m}(S)$ ) formed by arcs (respectively $m$-jets) in $S$ with origin in $Z$.

By a theorem of Greenberg [1966], $j_{m}(H(S))$ is a constructible subset of the $k$-variety $H_{m}(S)$ for any integer $m \geq 0$. We can then consider the class

$$
\left[j_{m}(H(S))\right] \in K_{0}\left(\operatorname{Var}_{k}\right) .
$$

Greenberg's result implies also that there is a ring formula $\psi_{m}$ over $k$, such that for any field $K$ containing $k$, the $k$-rational points of $H_{m}(S)$ that can be lifted to $K$-rational points of $H(S)$ correspond to the tuples satisfying $\psi_{m}$ in $K$. If $\psi_{m}^{\prime}$ is another ring formula over $k$ with the same property, then $\left[\psi_{m}\right]=\left[\psi_{m}^{\prime}\right]$ in $K_{0}\left(\right.$ Field $\left._{k}\right)$. The same applies for $j_{m}\left(H(S)_{Z}\right)$ if $Z \subset S$ is a closed subvariety.

Notation 2.1. We denote the class $\left[\psi_{m}\right]$ by $\left[j_{m}(H(S))\right]_{f}$ to avoid confusion with the class $\left[j_{m}(H(S))\right] \in K_{0}\left(\operatorname{Var}_{k}\right)$.

Definition 2.2 [DL 1999; 2001].

(1) The geometric motivic Poincaré series of $(S, Z)$ is

$$
P_{\text {geom }}^{(S, Z)}(T):=\sum_{m \geq 0} \chi_{c}\left(\left[j_{m}\left(H(S)_{Z}\right)\right]\right) T^{m} \in K_{0}^{\operatorname{mot}}\left(\operatorname{Var}_{k}\right) \otimes \mathbb{Q} \llbracket T \rrbracket .
$$

(2) The arithmetic motivic Poincaré series of $(S, Z)$ is

$$
P_{\mathrm{ar}}^{(S, Z)}(T):=\sum_{m \geq 0} \chi_{f}\left(\left[j_{m}\left(H(S)_{Z}\right)\right]_{f}\right) T^{m} \in K_{0}^{\mathrm{mot}}\left(\operatorname{Var}_{k}\right) \otimes \mathbb{Q} \llbracket T \rrbracket .
$$


Remark 2.3. In order to have the geometric and arithmetic setting in the same ring, we have slightly modified the original definition of the geometric motivic Poincaré series, since $\sum_{m \geq 0}\left[j_{m}\left(H(S)_{Z}\right)\right] T^{m} \in K_{0}\left(\operatorname{Var}_{k}\right) \llbracket T \rrbracket$; see [DL 1999]. This does not affect the rationality results below.

Denef and Loeser proved that these series have a rational form:

Theorem 2.4 [DL 1999, Theorem 1.1; 2001, Theorem 9.2.1]. The series $P_{\text {geom }}^{(S, Z)}(T)$ and $P_{\mathrm{ar}}^{(S, Z)}(T)$ belong to the subring of $K_{0}^{\mathrm{mot}}\left(\operatorname{Var}_{k}\right) \otimes \mathbb{Q} \llbracket T \rrbracket$ generated by

$$
K_{0}^{\mathrm{mot}}\left(\operatorname{Var}_{k}\right) \otimes \mathbb{Q}[T]
$$

and the series $\left(1-\mathbf{L}^{a} T^{b}\right)^{-1}$, with $a \in \mathbb{Z}$ and $b>0$.

The arithmetic motivic Poincaré series has interesting properties of specialization to classical arithmetic series. Let $p$ be a prime number. The operators $N_{p}$ and $N_{p, m}$ are applied to a variety $V$ defined over the integers by $N_{p}(V):=\# V(\mathbb{Z} / p \mathbb{Z})$ and $N_{p, m}(V):=\#\left\{\pi_{m}\left(V\left(\mathbb{Z}_{p}\right)\right)\right\}$, where $\mathbb{Z}_{p}$ denotes the $p$-adic integers,

$$
\pi_{m}\left(V\left(\mathbb{Z}_{p}\right)\right) \subset V\left(\mathbb{Z} / p^{m+1} \mathbb{Z}\right)
$$

is the image of $\left.V\left(\mathbb{Z}_{p}\right)\right)$ by the natural projection induced by $\mathbb{Z}_{p} \rightarrow \mathbb{Z} / p^{m+1} \mathbb{Z}$, and \# denotes the cardinality. Suppose that the variety $S$ is defined over the integers. The Serre-Oesterlé series $P_{p}^{S}(T):=\sum_{m \geq 0} N_{p, m} T^{m} \in \mathbb{Z} \llbracket T \rrbracket$ of $S$ at the prime $p$ is a rational function of $T$ [Denef 1984]. Denef and Loeser proved that for $p \gg 0$, the series $P_{p}^{S}(T)$ is obtained from $P_{\mathrm{ar}}^{S}(T)$ by applying to each coefficient the operator $N_{p}$ [DL 2001; 2002; 2004].

Remark 2.5. These results hold in a more general setting, in particular when $S$ is not affine as assumed here [DL 1999; 2001]. The proof of the rationality of $P_{p}^{S}(T)$ involves the use of quantifier elimination results and $p$-adic integration [Denef 1984]. The proof of the rationality of $P_{\mathrm{ar}}^{S}(T)$ requires also quantifier elimination results and arithmetic motivic integration [DL 2001; 2004; 2002].

\section{Affine toric varieties and monomial ideals}

In this section we introduce the basic notions and notations from toric geometry; see [Ewald 1996; Oda 1988; Fulton 1993; Gel'fand et al. 1994] for proofs.

If $N \cong \mathbb{Z}^{d}$ is a lattice, we denote by $N_{\mathbb{R}}:=N \otimes \mathbb{R}$ the vector space spanned by $N$ over the field $\mathbb{R}$, and by $N_{\mathbb{Q}}:=N \otimes \mathbb{Q}$ the vector space spanned by $N$ over $\mathbb{Q}$. In what follows, a cone in $N_{\mathbb{R}}$ means a rational convex polyhedral cone: the set of nonnegative linear combinations of vectors $a_{1}, \ldots, a_{r} \in N$. The cone $\tau$ is strictly convex if it contains no line through the origin, in which case we denote by 0 the 0 -dimensional face of $\tau$; the cone $\tau$ is simplicial if the primitive vectors of the 
1-dimensional faces are linearly independent over $\mathbb{R}$. We denote by $\tau$ or by $\operatorname{int}(\tau)$ the relative interior of the cone $\tau$.

We denote by $M$ the dual lattice. The dual cone $\tau^{\vee} \subset M_{\mathbb{R}}$ of $\tau$ is the set $\left\{w \in M_{\mathbb{R}} \mid\langle w, u\rangle \geq 0\right.$ for all $\left.u \in \tau\right\}$. The orthogonal cone $\tau^{\perp}$ has the condition $\langle w, u\rangle=0$ instead of $\langle w, u\rangle \geq 0$.

A fan $\Sigma$ is a family of strictly convex cones in $N_{\mathbb{R}}$ such that any face of such a cone is in the family and the intersection of any two of them is a face of each. The relation $\theta \leq \tau$ denotes that $\theta$ is a face of $\tau$. By $\theta<\tau$, we mean $\theta \neq \tau$ is a face of $\tau$. The support of the fan $\Sigma$ is the set $|\Sigma|:=\bigcup_{\tau \in \Sigma} \tau \subset N_{\mathbb{R}}$. The k-skeleton of the fan $\Sigma$ is $\Sigma^{(k)}=\{\tau \in \Sigma \mid \operatorname{dim} \tau=k\}$. We say that a fan $\Sigma^{\prime}$ is a subdivision of the fan $\Sigma$ if both fans have the same support and if every cone of $\Sigma^{\prime}$ is contained in a cone of $\Sigma$. If $\Sigma_{i}$ for $i=1, \ldots, n$ are fans with the same support, their intersection $\bigcap_{i=1}^{n} \Sigma_{i}:=\left\{\bigcap_{i=1}^{n} \tau_{i} \mid \tau_{i} \in \Sigma_{i}\right\}$ is also a fan.

Notation 3.1. In this paper, $\Lambda$ is a subsemigroup of finite type of a lattice $M$, which generates $M$ as a group and such that the cone $\sigma^{\vee}=\mathbb{R}_{\geq 0} \Lambda$ is strictly convex and of dimension $d$. We denote by $N$ the dual lattice of $M$ and by $\sigma \subset N_{\mathbb{R}}$ the dual cone of $\sigma^{\vee}$. We denote by $Z^{\Lambda}$ the affine toric variety $Z^{\Lambda}=\operatorname{Spec} k[\Lambda]$, where $k[\Lambda]=\left\{\sum_{\text {finite }} a_{\lambda} X^{\lambda} \mid a_{\lambda} \in k\right\}$ denotes the semigroup algebra of the semigroup $\Lambda$ with coefficients in the field $k$. The semigroup $\Lambda$ has a unique minimal set of generators $e_{1}, \ldots, e_{n}$; see the proof of [Ewald 1996, Lemma V.3.5, page 155]. We have an embedding of $Z^{\Lambda} \subset \mathbf{A}_{k}^{n}$ given by $x_{i}:=X^{e_{i}}$ for $i=1, \ldots, n$.

If $\Lambda=\sigma^{\vee} \cap M$, then the variety $Z^{\Lambda}$, which we denote also by $Z_{\sigma, N}$ or by $Z_{\sigma}$ when the lattice is clear from the context, is normal. If $\Lambda \neq \sigma^{\vee} \cap M$, the inclusion of semigroups $\Lambda \rightarrow \bar{\Lambda}:=\sigma^{\vee} \cap M$ defines a toric modification $Z^{\bar{\Lambda}} \rightarrow Z^{\Lambda}$, which is the normalization map.

The torus $T_{N}:=Z^{M}$ is an open dense subset of $Z^{\Lambda}$ that acts on $Z^{\Lambda}$, and the action extends the action of the torus on itself by multiplication. The origin 0 of the affine toric variety $Z^{\Lambda}$ is the 0 -dimensional orbit, defined by the maximal ideal $\left(X^{\lambda}\right)_{0 \neq \lambda \in \Lambda}$ of $k[\Lambda]$. There is a one-to-one inclusion reversing correspondence between the faces of $\sigma$ and the orbit closures of the torus action on $Z^{\Lambda}$. If $\theta \leq \sigma$, we denote by $\operatorname{orb}_{\theta}^{\Lambda}$ the orbit corresponding to the face $\theta$ of $\sigma$. The orbit closures are of the form $Z^{\Lambda \cap \theta^{\perp}}$ for $\theta \leq \sigma$.

Notation 3.2. The Newton polyhedron of a monomial ideal corresponding to a nonempty set of lattice vectors $\mathscr{I} \subset \Lambda$ is defined as the convex hull of the Minkowski sum of sets $\mathscr{I}+\sigma^{\vee}$. We denote this polyhedron by $\mathcal{N}(\mathscr{F})$. Notice that the vertices of $\mathcal{N}(\mathscr{Y})$ are elements of $\Phi$. We denote by ord $\Phi$ the support function of the polyhedron $\mathcal{N}(\mathscr{Y})$, which is defined by $\operatorname{ord}_{\mathscr{I}}: \sigma \rightarrow \mathbb{R}, v \mapsto \inf _{\omega \in \mathcal{N}(\mathscr{F})}\langle v, \omega\rangle$. A vector $v \in \sigma$ defines the face $\mathscr{F}_{\nu}:=\left\{\omega \in \mathcal{N}(\mathscr{I}) \mid\langle\nu, \omega\rangle=\operatorname{ord}_{\mathscr{T}}(\nu)\right\}$ of the polyhedron $\mathcal{N}(\mathscr{I})$. All faces of $\mathcal{N}(\mathscr{I})$ are of this form, and the compact faces are defined by vectors $v \in \stackrel{\circ}{\circ}$. 


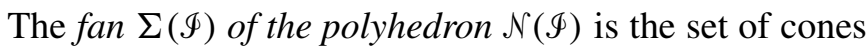

$$
\sigma(\mathscr{F}):=\left\{v \in \sigma \mid\langle\nu, \omega\rangle=\operatorname{ord}_{\mathscr{H}}(\nu) \text { for all } \omega \in \mathscr{F}\right\}
$$

for $\mathscr{F}$ running through the faces of $\mathcal{N}(\mathscr{Y})$. The fan $\Sigma(\mathscr{F})$ is supported on $\sigma$. By definition, it is easy to check that if $\tau \in \Sigma(\mathscr{I})$ and if $\nu, v^{\prime} \in \tau$, then the faces of the polyhedron $\mathcal{N}(\mathscr{I})$ defined by $v$ and $v^{\prime}$ coincide, that is, $\mathscr{F}_{v}=\mathscr{F}_{v^{\prime}}$; we denote this face also by $\mathscr{F}_{\tau}$.

The affine varieties $Z_{\tau}$ corresponding to cones $\tau$ in a fan $\Sigma$ glue up to define a toric variety $Z_{\Sigma}$. A fan $\Sigma$ subdividing the cone $\sigma$ defines a toric modification $\pi_{\Sigma}: Z_{\Sigma} \rightarrow Z_{\sigma}$.

If $\mathscr{\Im} \subset \Lambda$ defines a monomial ideal, the composite $Z_{\Sigma(\mathscr{F})} \stackrel{\pi_{\Sigma(\mathscr{F})}}{\longrightarrow} Z_{\sigma} \longrightarrow Z^{\Lambda}$ is equal to the normalized blowing up of $Z^{\Lambda}$ centered at $\mathscr{I}$; see for instance [Lejeune-Jalabert and Reguera 2003].

Definition 3.3. For $1 \leq j \leq n$, the $j$-th ideal of sums of combinations of $Z^{\Lambda}$ is the monomial ideal $\mathscr{C}_{j}$ of $k[\Lambda]$ generated by $X^{\alpha}$, where $\alpha$ runs through

$$
\left\{e_{i_{1}}+\cdots+e_{i_{j}} \mid\left\{i_{1}, \ldots, i_{j}\right\} \in\left(\begin{array}{c}
\{1, \ldots, n\} \\
j
\end{array}\right)\right\},
$$

where $\left(\begin{array}{c}\{1, \ldots, n\} \\ j\end{array}\right)$ denotes the set of combinations of $j$ elements of $\{1, \ldots, n\}$ for $j=1, \ldots, n$. We denote by $\Theta_{j}$ the fan of the polyhedron $\mathcal{N}\left(\mathscr{C}_{j}\right)$, and by $\operatorname{ord}_{\mathscr{C}_{j}}$ its support function; see Notation 3.2. The maps

$$
\varphi_{1}:=\operatorname{ord}_{\mathscr{C}_{1}} \quad \text { and } \quad \varphi_{j}:=\operatorname{ord}_{\mathscr{C}_{j}}-\operatorname{ord}_{C_{j-1}} \text { for } j=2, \ldots, n,
$$

are piecewise linear functions defined on the cone $\sigma$. If $v \in \sigma$, we write $\varphi_{0}(v):=0$ and $\varphi_{n+1}(\nu):=+\infty$ for convenience.

Definition 3.4. For $1 \leq l \leq d$, the $l$-th logarithmic jacobian ideal of $Z^{\Lambda}$ is the monomial ideal $\mathscr{g}_{l}$ of $k[\Lambda]$ generated by $X^{\alpha}$, where $\alpha$ runs through

$$
\left\{e_{i_{1}}+\cdots+e_{i_{l}} \mid e_{i_{1}} \wedge \cdots \wedge e_{i_{l}} \neq 0 \text { for } 1 \leq i_{1}, \ldots, i_{l} \leq n\right\} .
$$

We denote by $\Sigma_{l}$ the fan of the polyhedron $\mathcal{N}\left(\mathscr{F}_{l}\right)$, and by $\operatorname{ord}_{\mathscr{F}_{l}}$ its support function; see Notation 3.2. The maps

$$
\phi_{1}:=\operatorname{ord}_{\mathscr{F}_{1}} \quad \text { and } \quad \phi_{l}:=\operatorname{ord}_{\mathscr{F}_{l}}-\operatorname{ord}_{\mathscr{F}_{l-1}} \quad \text { for } l=2, \ldots, d,
$$

are piecewise linear functions defined on the cone $\sigma$. If $v \in \sigma$, we write $\phi_{0}(v):=0$ and $\phi_{d+1}(v):=+\infty$ for convenience.

We also use the notations $\mathscr{F}_{l}$ and $\mathscr{C}_{j}$ for the sets (4) and (3), respectively.

Lemma 3.5. If $v \in \circ$ and if $\left(p_{1}, \ldots, p_{n}\right)$ is a permutation of $(1, \ldots, n)$ such that

$$
\left\langle v, e_{p_{1}}\right\rangle \leq \cdots \leq\left\langle v, e_{p_{n}}\right\rangle,
$$


then $\operatorname{ord}_{C_{j}}(v)=\left\langle v, \sum_{r=1}^{j} e_{p_{r}}\right\rangle$ and $\varphi_{j}(v)=\left\langle v, e_{p_{j}}\right\rangle$ for $1 \leq j \leq n$. Moreover,

$$
0=\varphi_{0}(v) \leq \varphi_{1}(v) \leq \cdots \leq \varphi_{n}(v) \quad \text { and } \quad 0=\phi_{0}(v) \leq \phi_{1}(v) \leq \cdots \leq \phi_{d}(v) .
$$

Proof. The first assertion follows by induction on $j \in\{1, \ldots, n\}$.

See [Cobo Pablos and González Pérez 2012, Lemma 5.3] for the second sequence of inequalities.

Proposition 3.6. The Newton polyhedra of the ideals $\mathscr{b}_{j}$ for $j=1, \ldots, n$ determine and are determined by the minimal system of generators of the semigroup $\Lambda$.

Proof. The Newton polyhedron $\mathcal{N}\left(\mathscr{C}_{j}\right)$ determines and is determined by its support function $\operatorname{ord}_{\mathfrak{C}_{j}}$ for $j=1, \ldots, n$. By Lemma 3.5 and the definitions if $\theta$ is a $d$-dimensional cone of the fan $\bigcap_{r=1}^{n} \Theta_{r}$, there exists a permutation $i_{1}, \ldots, i_{n}$ of $1, \ldots, n$ such that $\varphi_{j}(v)=\left\langle v, e_{i_{j}}\right\rangle$ for $j=1, \ldots, n$ and all $v \in \stackrel{\circ}{ }$. Thus, the functions $\varphi_{j}$ for $j=1, \ldots, n$ or equivalently, $\operatorname{ord}_{\mathscr{C}_{j}}$ for $j=1, \ldots, n$ determine the vectors $e_{1}, \ldots, e_{n}$.

\section{Arcs and jets on a toric singularity}

Let $\Lambda$ be a semigroup as in Notation 3.1. If $R$ is a $k$-algebra, an $R$-rational point of $Z^{\Lambda}$ is a homomorphism of semigroups $(\Lambda,+) \rightarrow(R, \cdot)$, where $(R, \cdot)$ denotes the semigroup $R$ for the multiplication. In particular, the closed points are obtained for $R=k$. An $\operatorname{arc} h$ on the affine toric variety $Z^{\Lambda}$ is given by a semigroup homomorphism $(\Lambda,+) \rightarrow(k \llbracket t \rrbracket, \cdot)$. An arc in the torus $T_{N}$ is defined by a semigroup homomorphism $\Lambda \rightarrow k \llbracket t \rrbracket^{*}$, where $k \llbracket t \rrbracket^{*}$ denotes the group of units of the ring $k \llbracket t \rrbracket$.

Notation 4.1. We denote the set of $\operatorname{arcs} H\left(Z^{\Lambda}\right)_{0}$ of $Z^{\Lambda}$ with origin at the distinguished point 0 of $Z^{\Lambda}$ simply by $H_{\Lambda}$, and by $H_{\Lambda}^{*}$ the set consisting of those arcs of $H_{\Lambda}$ with generic point in the torus $T_{N}$.

Notice that $h \in H_{\Lambda}^{*}$ if and only if the formal power series $X^{u} \circ h \in k \llbracket t \rrbracket$ is nonzero for all $u \in \Lambda$. Any arc $h \in H_{\Lambda}^{*}$ defines two group homomorphisms $v_{h}: M \rightarrow \mathbb{Z}$ and $\omega_{h}: M \rightarrow k \llbracket t \rrbracket^{*}$ by $X^{m} \circ h=t^{v_{h}(m)} \omega_{h}(m)$. If $m \in \Lambda$, then $v_{h}(m)>0$, and hence $v_{h}$ belongs to $\stackrel{\circ}{\cap} \cap N$. Notice that $\omega_{h}$ defines an arc in the torus, that is, $\omega_{h} \in H\left(T_{N}\right)$.

Remark 4.2. The space of arcs in the torus acts on the arc space of a toric variety; [Ishii 2004; 2005].

Lemma 4.3 [Ishii 2004, Theorem 4.1; 2005, Lemma 5.6; Lejeune-Jalabert and Reguera 2003, Proposition 3.3]. The map $\circ \cap N \times H\left(T_{N}\right) \rightarrow H_{\Lambda}^{*}$, which applies a pair $(v, \omega)$ to the arc $h$ defined by $X^{u} \circ h=t^{\langle\nu, u\rangle} \omega(u)$ for $u \in \Lambda$ is a bijection. The sets $H_{\Lambda, v}^{*}:=\left\{h \in H_{\Lambda}^{*} \mid v_{h}=v\right\}$ for $v \in \circ \cap N$ are orbits for the action of $H\left(T_{N}\right)$ on $H_{\Lambda}^{*}$, and we have $H_{\Lambda}^{*}=\bigsqcup_{v \in \delta \cap \cap N} H_{\Lambda, v}^{*}$. 
Remark 4.4. We often denote the set $H_{\Lambda}^{*}$ and orbit $H_{\Lambda, v}^{*}$ by $H^{*}$ and $H_{v}^{*}$, respectively, if $\Lambda$ is clear from the context.

An arc $h \in H_{\Lambda}$ has its generic point $\eta$ contained in exactly one orbit of the torus action on $Z^{\Lambda}$. If $h(\eta) \in \operatorname{orb}_{\theta}^{\Lambda}$ for some $\theta \leq \sigma$, then $h$ factors through the orbit closure $Z^{\Lambda \cap \theta^{\perp}}$ and $h \in H_{\Lambda \cap \theta^{\perp}}^{*}$, that is, $h$ is an arc through $\left(Z^{\Lambda \cap \theta^{\perp}}, 0\right)$ with generic point in the torus $\operatorname{orb}_{\theta}^{\Lambda}$. We can apply Lemma 4.3 to describe the set $H_{\Lambda \cap \theta^{\perp}}^{*}$, just replacing the semigroup $\Lambda$ by $\Lambda \cap \theta^{\perp}$; see [Cobo Pablos and González Pérez 2012]. In particular, if $\theta=0$, then $h \in H_{\Lambda}^{*}$; if $\theta=\sigma$, then $\Lambda \cap \theta^{\perp}=0$ and $h$ is the constant arc at the distinguished point $0 \in Z^{\Lambda}$. We have a partition $H_{\Lambda}=\bigsqcup_{\theta \leq \sigma} H_{\Lambda \cap \theta^{\perp}}^{*}$.

\section{The image of the class of the formula defining $j_{s}\left(H_{v}^{*}\right)$}

Definition 5.1. We associate to $(v, s) \in(\circ \cap N) \times \mathbb{Z}_{>0}$ the sets

$$
\begin{aligned}
M_{v}^{s} & :=\operatorname{span}_{\mathbb{Z}}\left\{e_{i} \mid\left\langle v, e_{i}\right\rangle \leq s, i=1, \ldots, n\right\}, \\
\ell_{v}^{s} & :=\operatorname{span}_{\mathbb{Q}}\left\{e_{i} \mid\left\langle v, e_{i}\right\rangle \leq s, i=1, \ldots, n\right\} .
\end{aligned}
$$

We denote by $l(v, s)$ the dimension of the $\mathbb{Q}$-vector space $\ell_{v}^{s}$. The integer $l(v, s)$ is also the rank of the lattice $M_{v}^{s}$. We denote by $q(v, s)$ the index of the lattice extension $M_{v}^{s} \subset \ell_{v}^{s} \cap M$.

Proposition 5.2. If $(v, s) \in \stackrel{\circ}{\sigma} \times \mathbb{Z}_{>0}$, with $l(v, s)>0$, and if the field $k$ contains all the $q(v, s)$-th roots of unity, then we have

$$
\chi_{f}\left(\left[j_{s}\left(H_{v}^{*}\right)\right]_{f}\right)=\frac{1}{q(v, s)}(\mathbf{L}-1)^{l(v, s)} \times \mathbf{L}^{s l(v, s)-\operatorname{ord}_{\mathscr{f}_{l(v, s)}}(v)} .
$$

If $l(v, s)=0$, then we have $\chi_{f}\left(\left[j_{s}\left(H_{v}^{*}\right)\right]_{f}\right)=1$.

Proof. If $h \in H_{v}^{*}$, the equality $\operatorname{ord}_{t}\left(X^{e_{i}} \circ h\right)=\left\langle\nu, e_{i}\right\rangle$ holds for $1 \leq i \leq n$. By Definition 5.1, those vectors $e_{i}$ such that $j_{s}\left(X^{e_{i}} \circ h\right) \neq 0$ span the $\mathbb{Q}$-vector space $\ell_{v}^{s}$, since $\left\langle v, e_{i}\right\rangle \leq s$. If $l(v, s)=0$, this vector space is empty, the jet space $j_{s}\left(H_{v}^{*}\right)$ consists of the constant 0 -jet, and the conclusion follows easily from the definitions.

Suppose then that $l:=l(v, s)>0$. If $h \in H_{v}^{*}$, then it is given by $n$ series of the form

$$
X^{e_{i}} \circ h=t^{\left\langle\nu, e_{i}\right\rangle} c\left(e_{i}\right)\left(1+\sum_{m \geq 1} u_{m}\left(e_{i}\right) t^{m}\right) \quad \text { for } i=1, \ldots, n .
$$

The $s$-jet $j_{s}\left(X^{e_{i}} \circ h\right)$ is different from zero if and only if $\left\langle v, e_{i}\right\rangle \leq s$.

By [Cobo Pablos and González Pérez 2012, Lemma 5.7], there exist integers $1 \leq k_{1}, \ldots, k_{l} \leq n$ such that $\phi_{i}(v)=\left\langle v, e_{k_{i}}\right\rangle \leq s$ for $i=1, \ldots, l$,

$$
\ell_{v}^{s}=\operatorname{span}_{\mathbb{Q}}\left\{e_{k_{1}}, \ldots, e_{k_{l}}\right\}, \quad \text { and } \quad \operatorname{ord}_{\mathscr{F}_{l}}(v)=\sum_{i=1}^{l}\left\langle v, e_{k_{i}}\right\rangle .
$$


By [Cobo Pablos and González Pérez 2012, Section 6], if $h$ is the universal family of arcs parametrizing $H_{v}^{*}$, the terms $\left\{u_{m}\left(e_{k_{i}}\right) \mid i=1, \ldots, l, m \geq 1\right\}$ are algebraically independent over $\mathbb{Q}$ and the terms $\left\{c\left(e_{i}\right)^{ \pm 1} \mid i=1, \ldots, n\right\}$ generate a $k$-algebra isomorphic to $k[M]$ by the isomorphism that maps $c\left(e_{i}\right) \mapsto X^{e_{i}}$.

By the proof of [Cobo Pablos and González Pérez 2012, Theorem 7.1], a formula defining $j_{s}\left(H_{v}^{*}\right)$ is the conjunction of two formulas, $\psi_{1}$ and $\psi_{2}$, with independent sets of variables. The first formula, $\psi_{1}$, is a finite sequence of polynomial equalities with rational coefficients expressing the terms $u_{r}\left(e_{i}\right)$ appearing in $j_{s}\left(X^{e_{i}} \circ h\right)$, for $1 \leq r \leq s-\left\langle v, e_{i}\right\rangle$, in terms of the variables

$$
\left\{u_{r}\left(e_{k_{i}}\right) \mid 1 \leq i \leq l, 1 \leq r \leq s-\left\langle v, e_{k_{i}}\right\rangle\right\} .
$$

We deduce that $\chi_{f}\left(\left[\psi_{1}\right]\right)=\mathbf{L}^{s l-\operatorname{ord}_{q_{l}}(v)}$. The second formula comes from the effect on the initial coefficients $c\left(e_{i}\right)$ for $e_{i} \in \ell_{v}^{s}$ of the operation taking the $s$-jet of an arc. This operation is described by taking the image by the map

$$
\Psi: T^{\prime}:=\operatorname{Spec} k\left[c\left(e_{i}\right)^{ \pm 1}\right]_{e_{i} \in \ell_{v}^{s}} \rightarrow T:=\operatorname{Spec} k\left[c\left(e_{i}\right)^{ \pm 1}\right]_{\left\langle v, e_{i}\right\rangle \leq s}
$$

of the point determined by $h \in H_{v}^{*}$. The map $\Psi$ is the unramified covering of $l$-dimensional algebraic tori determined by the inclusion $M_{v}^{s} \subset \ell_{v}^{s} \cap M$ of index $q(\nu, s)$ of rank $l(v, s)$ lattices. Thus the second formula is equivalent to $\psi_{2}$ : there exists $y \in T^{\prime}$ such that $\Psi(y)=x$ for $x \in T$, and hence by Lemma 1.2 we get that $\chi_{f}\left(\left[\psi_{2}\right]\right)=(1 / q(\nu, s))(\mathbf{L}-1)^{l}$.

\section{Sequences of convex piecewise linear functions and fans}

Let $\sigma \subset N_{\mathbb{R}}$ be a rational convex polyhedral cone of dimension $d=\operatorname{dim} N_{\mathbb{R}}$. Consider a sequence of piecewise linear continuous functions

$$
h_{p}: \sigma \rightarrow \mathbb{R} \text { for } 1 \leq p \leq m,
$$

such that $h_{p}(\sigma \cap N) \subset \mathbb{Z}$, and

$$
0 \leq h_{1}(v) \leq \cdots \leq h_{m}(v) \text { for all } v \in \sigma .
$$

By convenience we set $h_{0}(v)=0$ and $h_{m+1}(\nu)=+\infty$. We denote by $\Xi_{0}$ the fan consisting on the faces of $\sigma$ and by $\Xi_{p}$ the coarser fan such that the restriction of $h_{p}$ to $\eta$ is linear for any cone $\eta \in \Xi_{p}$ for $1 \leq p \leq m$. In addition we assume that for any cone $\eta \in \Xi_{p-1}$ the restriction $h_{p \mid \eta}$ is upper convex, that is, $h_{p}(v)+h_{p}\left(v^{\prime}\right) \leq$ $h_{p}\left(v+v^{\prime}\right)$ for all $v, v^{\prime} \in \eta$.

Notation 6.1. For $0 \leq p \leq m$ and for $\eta \in \bigcap_{r=1}^{p} \Xi_{r}$ we set

$$
\eta(\underline{h}, p):=\left\{(v, s) \in N_{\mathbb{R}} \times \mathbb{R}_{\geq 0} \mid v \in \stackrel{\circ}{ } \cap \stackrel{\circ}{ }, h_{p}(v) \leq s<h_{p+1}(v)\right\} .
$$


Lemma 6.2. The closure $\bar{\eta}(\underline{h}, p)$ of the set $\eta(\underline{h}, p)$ is a convex polyhedral cone that is rational for the lattice $N \times \mathbb{Z}$.

Proof. If $\eta \in \bigcap_{r=0}^{p} \Xi_{r}$, then the restriction $h_{j \mid \eta}: \eta \rightarrow \mathbb{R}$ is linear if $j=p$, and upper convex if $j=p+1$. It follows that $\bar{\eta}(\underline{h}, p)$ is a convex polyhedral cone, rational for the lattice $N \times \mathbb{Z}$, since $h_{p}$ and $h_{p+1}$ take integral values on $N$.

Notation 6.3. For $0 \leq p \leq m$ and $\eta \in \boldsymbol{\Xi}_{p}$ we define the following sets:

(i) $A(\underline{h}, p):=\left\{(v, s) \in N \times \mathbb{Z} \mid v \in \stackrel{\circ}{ }, h_{p}(v) \leq s<h_{p+1}(v)\right\}$.

(ii) $A(\underline{h}, p, \eta):=\left\{(v, s) \in N \times \mathbb{Z} \mid v \in \stackrel{\circ}{\cap} \stackrel{\circ}{ }, h_{p}(v) \leq s<h_{p+1}(v)\right\}$.

Remark 6.4. We have partitions

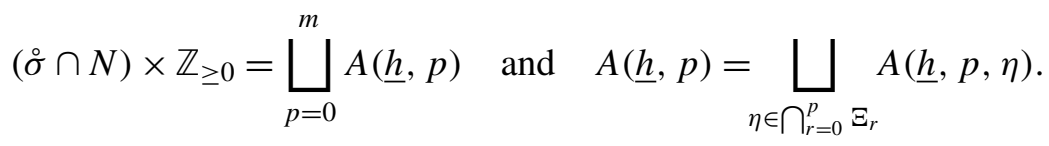

\section{Refinements of partitions}

We apply the procedure of Section 6 to both sequences $\underline{\phi}=\left(\phi_{1}, \ldots, \phi_{d}\right)$ and $\underline{\varphi}=\left(\varphi_{1}, \ldots, \varphi_{n}\right)$ (see Lemma 3.5).

Remark 7.1. The sequence of fans associated to $\underline{\phi}$ (respectively $\underline{\varphi}$ ) is $\bigcap_{r=0}^{i} \Sigma_{r}$ for $i=0, \ldots, d$ (respectively $\bigcap_{r=0}^{i} \Theta_{r}$ for $i=0, \ldots, n$ ), where for convenience we denote by $\Sigma_{0}$ or by $\Theta_{0}$ the fan consisting of the faces of the cone $\sigma$.

Lemma 7.2. If $A(\underline{\varphi}, j, \theta) \neq \varnothing$ for some $1 \leq j \leq n$ and $\theta \in \bigcap_{r=1}^{j} \Theta_{r}$ (using Notation 6.3), then the restriction of the functions $(\circ \cap N) \times \mathbb{Z}_{>0} \rightarrow \mathbb{Z}_{\geq 0}$ given by

$$
(\nu, s) \mapsto l(v, s) \quad \text { and } \quad(\nu, s) \mapsto q(\nu, s)
$$

to the set $A(\varphi, j, \theta)$ are constant functions. We denote their values on the set $A(\underline{\varphi}, j, \theta)$ by $\bar{l}(j, \theta)$ and $q(j, \theta)$, respectively.

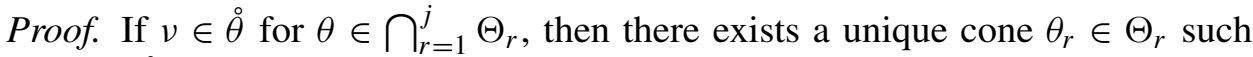
that $v \in \dot{\theta}_{r}$ for $r=1, \ldots, j$. We denote by $\mathscr{F}_{r, \theta}$ the face of the polyhedron $\mathcal{N}\left(\mathscr{C}_{r}\right)$ defined by any such vector $v \in \grave{\theta}$ for $r=1, \ldots, j$ (see Notation 3.2).

Suppose that $v, v^{\prime} \in \stackrel{\circ}{ }$ and $\left(p_{1}, \ldots, p_{n}\right)$ and $\left(p_{1}^{\prime}, \ldots, p_{n}^{\prime}\right)$ are two permutations of $(1, \ldots, n)$ such that the inequalities

$$
\left\langle v, e_{p_{1}}\right\rangle \leq \cdots \leq\left\langle v, e_{p_{n}}\right\rangle \quad \text { and } \quad\left\langle v^{\prime}, e_{p_{1}^{\prime}}\right\rangle \leq \cdots \leq\left\langle v^{\prime}, e_{p_{n}^{\prime}}\right\rangle
$$

hold. We prove first that

$$
\left\langle v, e_{p_{1}^{\prime}}\right\rangle \leq \cdots \leq\left\langle v, e_{p_{j}^{\prime}}\right\rangle .
$$


By definition, for any $1 \leq r \leq j$, we have that $\operatorname{ord}_{\mathscr{C}_{r}}(v)=\left\langle v, u_{r}\right\rangle$ for any $u_{r} \in \mathscr{F}_{r, \theta}$. We get from Lemma 3.5 that the vectors $u_{r}:=e_{p_{1}}+\cdots+e_{p_{r}}$ and $u_{r}^{\prime}:=e_{p_{1}^{\prime}}+\cdots+e_{p_{r}^{\prime}}$ belong to $\mathscr{F}_{r, \theta}$ for $1 \leq r \leq j$. This implies (7).

If $(v, s) \in A(\underline{\varphi}, j, \theta)$, then by Lemma 3.5, we obtain $\varphi_{j}(v)=\left\langle v, e_{p_{j}}\right\rangle \leq s<$ $\varphi_{j+1}(v)$. We deduce that if $(v, s)$ and $\left(v^{\prime}, s^{\prime}\right)$ belong to $A(\underline{\varphi}, j, \theta)$, then

$$
\left\{e_{i} \mid 1 \leq i \leq n,\left\langle v, e_{i}\right\rangle \leq s\right\}=\left\{e_{i} \mid 1 \leq i \leq n,\left\langle v^{\prime}, e_{i}\right\rangle \leq s^{\prime}\right\}=\left\{e_{p_{1}}, \ldots, e_{p_{j}}\right\} .
$$

Since (8) spans the lattice $M_{v}^{s}$ and the vector space $\ell_{v}^{s}$, the sublattices $\ell_{v}^{s} \cap M$ and $M_{v}^{s}$ are independent of the choice of $(v, s)$ in $A(\underline{\varphi}, j, \theta)$. This implies the constancy of the functions $l$ and $q$ on $A(\underline{\varphi}, j, \theta)$.

Remark 7.3. If $1 \leq l \leq d$ and if $\tau \in \bigcap_{r=1}^{l} \Sigma_{r}$, we denoted in [Cobo Pablos and González Pérez 2012] the sets $A(\phi, l)$ and $A(\phi, l, \tau)$ by $A_{l}$ and $A_{l, \tau}$, respectively. The map $l(v, s)$ is also constant on the sets of the form $A(\phi, l, \tau)$ for $\tau \in \bigcap_{i=0}^{l} \Sigma_{i}$; see [Cobo Pablos and González Pérez 2012, Lemma 5.7].

By Remark 6.4, we have two partitions

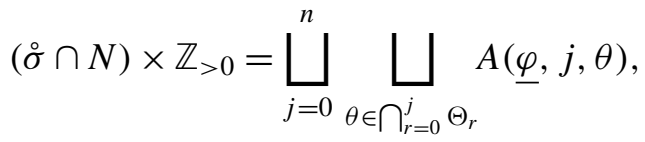

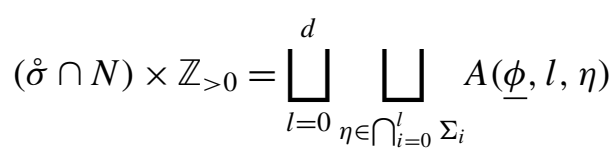

associated to the sequences $\underline{\varphi}$ and $\phi$.

Proposition 7.4. If $\theta(\underline{\varphi}, j) \neq \varnothing$ for some $1 \leq j \leq n$ and $\theta \in \bigcap_{r=1}^{j} \Theta_{r}$, then there exists a unique cone $\tau \in \bigcap_{r=1}^{l(j, \theta)} \Sigma_{r}$ such that $\theta \subset \tau$ and

$$
A(\underline{\varphi}, j, \theta) \subset A(\underline{\phi}, l(j, \theta), \tau) .
$$

Proof. Given $(v, s)$ and $\left(v^{\prime}, s^{\prime}\right)$ in $A(\underline{\varphi}, j, \theta) \subset(\stackrel{\circ}{\sigma} \cap N) \times \mathbb{Z}_{>0}$, we deduce from (9) that there exist cones $\tau \in \bigcap_{i=0}^{l} \Sigma_{i}$ and $\tau^{\prime} \in \bigcap_{i=0}^{l^{\prime}} \Sigma_{i}$ for integers $0 \leq l, l^{\prime} \leq d$ such that $(v, s) \in A(\phi, l, \tau)$ and $\left(v^{\prime}, s^{\prime}\right) \in A\left(\phi, l^{\prime}, \tau^{\prime}\right)$. By [Cobo Pablos and González Pérez 2012, Lemma 5.7], we have $l=l(\bar{v}, s)$ and $l^{\prime}=l\left(v^{\prime}, s^{\prime}\right)$, and then $l=l^{\prime}$ by (8). Then $l=l(j, \theta)$ by definition in Lemma 7.2.

Let $\left(p_{1}, \ldots, p_{n}\right)$ and $\left(p_{1}^{\prime}, \ldots, p_{n}^{\prime}\right)$ be two permutations of $(1, \ldots, n)$ such that (6) holds. Then we can apply the method given in [Cobo Pablos and González Pérez 2012, Proposition 5.1] to determine the value of $\operatorname{ord}_{q_{i}}(v)$ for $1 \leq i \leq l(v, s)$. Moreover, it is enough to apply this on the set (8) instead of $\left\{e_{1}, \ldots, e_{n}\right\}$. We deduce from (7) that $v$ and $v^{\prime}$ define the same face of $\mathcal{N}\left(\mathscr{F}_{i}\right)$ for $1 \leq i \leq l(v, s)$. This is equivalent to the equality $\tau=\tau^{\prime}$. We have proven (10), and as a consequence, the inclusion $\theta \subset \tau$ holds. 
Definition 7.5 [Cobo Pablos and González Pérez 2012, Definition 8.1 and Re-

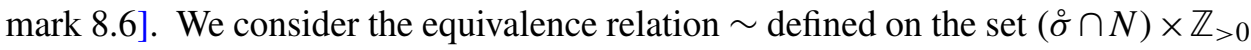
by

$$
(v, s) \sim\left(v^{\prime}, s^{\prime}\right) \Longleftrightarrow s=s^{\prime}, \ell_{v}^{s}=\ell_{v^{\prime}}^{s} \text { and } v_{\mid \ell_{v}^{s}}=v_{\mid \ell_{v^{\prime}}^{s}}^{\prime} .
$$

Lemma 7.6. The set $A(\varphi, j, \theta)$ is a union of equivalence classes by the relation $\sim$ of Definition 7.5 for $1 \leq \bar{j} \leq n$ and $\theta \in \bigcap_{r=1}^{j} \Theta_{r}$. Moreover, we have

$$
A(\underline{\phi}, l, \tau) / \sim=\bigsqcup_{\theta \in \bigcap_{r=1}^{j} \Theta_{r}, l(j, \theta)=l}^{\theta \subset \tau} A(\underline{\varphi}, j, \theta) / \sim .
$$

Proof. By (9) and Proposition 7.4, it follows that

$$
A(\underline{\phi}, l, \tau)=\bigsqcup_{\theta \in \bigcap_{r=1}^{j} \Theta_{r}, l(j, \theta)=l}^{\theta \subset \tau} A(\underline{\varphi}, j, \theta) .
$$

If $(v, s)$ belongs to $A(\varphi, j, \theta)$ and $(v, s) \sim\left(v^{\prime}, s\right)$, then (8) holds. The vectors $v$ and $v^{\prime}$ define the same face of $\mathcal{N}\left(\mathscr{C}_{r}\right)$ for $1 \leq r \leq j$, and therefore $v^{\prime} \in \operatorname{int} \theta$. Since $\varphi_{j}\left(v^{\prime}\right) \leq s<\varphi_{j+1}\left(v^{\prime}\right)$, we conclude that $\left(v^{\prime}, s\right) \in A(\underline{\varphi}, j, \theta)$.

\section{The structure of the series $P_{\mathrm{ar}}^{\left(Z^{\Lambda}, 0\right)}(T)$}

We consider the auxiliary Poincaré series

$$
P_{\mathrm{ar}}(\Lambda):=\sum_{s \geq 0} \chi_{f}\left(\left[j_{s}\left(H_{\Lambda}\right) \backslash \bigcup_{0 \neq \theta \leq \sigma} j_{s}\left(H_{\Lambda \cap \theta^{\perp}}\right)\right]_{f}\right) T^{s} \in K_{0}^{\mathrm{mot}}\left(\operatorname{Var}_{k}\right) \otimes \mathbb{Q} \llbracket T \rrbracket .
$$

Notice that the Poincaré series $P_{\text {ar }}(\Lambda)$ measures the class of the formula defining the set of jets of arcs with origin in 0 that are not jets of arcs factoring through proper orbit closures of the toric variety $Z^{\Lambda}$.

Proposition 8.1. We have $P_{\mathrm{ar}}^{\left(Z^{\Lambda}, 0\right)}(T)=\sum_{\theta \leq \sigma} P_{\mathrm{ar}}\left(\Lambda \cap \theta^{\perp}\right)$.

It follows from Proposition 8.1 that in order to describe the motivic series $P_{\mathrm{ar}}^{\left(Z^{\Lambda}, 0\right)}(T)$, it is enough to describe the form of the auxiliary series $P_{\mathrm{ar}}(\Lambda)$ for any semigroup $\Lambda$.

Remark 8.2. In the normal case, the equality $j_{m}\left(H_{\Lambda}\right)=j_{m}\left(H_{\Lambda}^{*}\right)$ holds for all $m \geq 0$ [Nicaise 2005b], but this property fails in general.

Definition 8.3 [Cobo Pablos and González Pérez 2012, Definition 8.9]. If $1 \leq l \leq d$, $\tau \in \bigcap_{i=1}^{l} \Sigma_{i}$, and $v \in \tau$, then $v$ defines a face $\mathscr{F}_{l, v}$ of the polyhedron $\mathcal{N}\left(\mathscr{F}_{l}\right)$. Since the face $\mathscr{F}_{l, v}$ is independent of the choice of $v \in \tau$, we denote it by $\mathscr{F}_{l, \tau}$. If $1 \leq l \leq d$, 
the set $\mathscr{D}_{l}$ is the subset of cones $\tau \in \bigcap_{i=1}^{l} \Sigma_{i}$ such that the face $\mathscr{F}_{l, \tau}$ of $\mathcal{N}\left(\mathscr{F}_{l}\right)$ is contained in the interior of $\sigma^{\vee}$. We denote by $\left|\mathscr{D}_{l}\right|$ the set $\bigcup_{\tau \in \mathscr{D}_{l}} \tau$.

Proposition 8.4. Let us fix an integer $s_{0} \geq 1$. The set $j_{s_{0}}\left(H_{\Lambda}^{*}\right) \backslash \bigcup_{0 \neq \theta \leq \sigma} j_{s_{0}}\left(H_{\Lambda \cap \theta^{\perp}}\right)$ expresses as a finite disjoint union of locally closed subsets, as follows:

$$
j_{s_{0}}\left(H_{\Lambda}^{*}\right) \backslash \bigcup_{0 \neq \theta \leq \sigma} j_{s_{0}}\left(H_{\Lambda \cap \theta^{\perp}}\right)=\bigsqcup_{j=1}^{n} \bigsqcup_{\theta \in \bigcap_{r=1}^{j} \Theta_{r}}^{\theta \subset \mid\left(\mathscr{D}_{l(j, \theta) \mid}\right.} \bigsqcup_{\left.\left(v, s_{0}\right)\right] \in A(\underline{\varphi}, j, \theta) / \sim} j_{s_{0}}\left(H_{\Lambda, \nu}^{*}\right) .
$$

Proof. This partition follows from the partition in [Cobo Pablos and González Pérez 2012, Proposition 8.11] by using formula (11) (see Remark 7.3).

If $s_{0} \geq 1$, the coefficient of $T^{s_{0}}$ in the auxiliary series $P(\Lambda)$ is obtained by applying the map $\chi_{f}$ to the class of the formula defining (13). Then we determine this class by using Proposition 5.2.

We introduce the following auxiliary series for $\theta \in \bigcap_{r=1}^{j} \Theta_{r}$ :

$$
P_{\underline{\varphi}, j, \theta}(\Lambda):=(\mathbf{L}-1)^{l(j, \theta)} \sum_{s \geq 1} \sum_{[(\nu, s)] \in A(\underline{\varphi}, j, \theta) / \sim} \mathbf{L}^{l(j, \theta) s-\operatorname{ord}_{\mathscr{F}_{l(j, \theta)}}(\nu)} T^{s} .
$$

We deduce the next proposition from Propositions 8.4 and 5.2 and formula (14). Proposition 8.5. $\quad P_{\mathrm{ar}}(\Lambda)=\sum_{j=1}^{n} \sum_{\theta \in \bigcap_{r=1}^{j} \Theta_{r}}^{\theta \subset \mid \mathscr{Q}_{l(j, \theta) \mid}} \frac{1}{q(j, \theta)} P_{\underline{\varphi}, j, \theta}(\Lambda)$.

\section{The rational form of some generating series}

In this section, we fix an integer $1 \leq j \leq n$ and a cone $\theta \in \bigcap_{r=1}^{j} \Theta_{r}$ such that $A(\underline{\varphi}, j, \theta) \neq \varnothing$. For simplicity, we denote by $l$ the integer $l(j, \theta)$ defined in Lemma 7.2 and by $\tau$ the unique element of the fan $\bigcap_{r=1}^{l} \Sigma_{r}$ such that (10) holds.

Since $\theta \subset \tau \subset \bigcap_{r=1}^{l} \Sigma_{r}$, the restriction of $\phi_{r}$ to $\theta$ is a linear function of the form

$$
\left(\phi_{r}\right)_{\mid \theta}(v)=\left\langle\nu, e_{i_{r}}\right\rangle \text { for } r=1, \ldots, l,
$$

where $\left\{i_{1}, \ldots, i_{l}\right\} \subset\{1, \ldots, n\}$.

Consider the lattice homomorphisms

$$
\mu: N \times \mathbb{Z} \rightarrow \mathbb{Z}^{l+1}, \quad(\nu, s) \mapsto\left(\left\langle v, e_{i_{1}}\right\rangle, \ldots,\left\langle\nu, e_{i_{l}}\right\rangle, s\right)
$$

and

$$
\pi=\left(\pi_{1}, \pi_{2}\right): \mathbb{Z}^{l+1} \rightarrow \mathbb{Z}^{2}, \quad\left(a_{1}, \ldots, a_{l+1}\right) \mapsto\left(l a_{l+1}-a_{1}-\cdots-a_{l}, a_{l+1}\right) .
$$

We set $\xi=\pi \circ \mu$. 
Remark 9.1. The homomorphisms $\pi, \mu$, and $\xi$ were considered in [Cobo Pablos and González Pérez 2012]. Since $\theta$ is contained in $\tau$, the kernels of $\mu$ and $\xi$ intersect the cone $\theta$ only at the origin. Similarly, by formula (10), the inclusion $\xi(A(\underline{\varphi}, j, \theta)) \subset \mathbb{Z}_{\geq 0}^{2} \backslash\{(0,0)\}$ holds. See [Cobo Pablos and González Pérez 2012, Section 9].

If $j \neq n$, the lower boundary of the cone $\theta$ is the set

$$
\partial_{-} \theta(\underline{\varphi}, j):=\left\{(v, s) \mid v \in \theta, s=\varphi_{j}(v)\right\} .
$$

Notice that $\partial_{-} \theta(\underline{\varphi}, j)$ is a cone since $\theta \in \bigcap_{r=1}^{j} \Theta_{j}$, and then the function $\varphi_{j}$ is linear on $\theta$. The upper boundary is the set

$$
\partial_{+} \theta(\underline{\varphi}, j):=\left\{(v, s) \mid v \in \theta, s=\varphi_{j+1}(v) \neq \varphi_{j}(v)\right\} .
$$

If $j=n$, then $l=d$ and $\varphi_{n+1}(v)=+\infty$, and the upper boundary is the union of cones spanned by $(0,1) \in N_{\mathbb{R}} \times \mathbb{R}$ and the proper faces of the cone $\partial_{-} \theta(\underline{\varphi}, j)$. The edges of the cone $\theta(\underline{\varphi}, j)$ are edges of $\partial_{-} \theta(\underline{\varphi}, j) \cup \partial_{+} \theta(\underline{\varphi}, j)$.

Notation 9.2. If $\rho \subset \tau$ is a one-dimensional cone rational for the lattice $N$, we denote by $v_{\rho}$ the primitive integral vector on $\rho$, that is, the generator of the semigroup $\rho \cap N$.

Remark 9.3. The primitive integral vectors for the lattice $N \times \mathbb{Z}$ on the edges of the cone $\theta$ are

$$
\left(v_{\rho}, \varphi_{j}\left(v_{\rho}\right)\right) \text { for } \rho \leq \theta, \operatorname{dim} \rho=1
$$

together with

$$
\begin{cases}(0,1) & \text { if } j=n \\ \left(v_{\rho}, \varphi_{j+1}\left(v_{\rho}\right)\right) & \text { if } j \neq n\end{cases}
$$

for $\rho \in \Theta_{j+1}, \rho \subset \theta, \operatorname{dim} \rho=1$, and $\varphi_{j}(v) \neq \varphi_{j+1}(v)$. Then notice that

$$
\xi(v, s)= \begin{cases}\left(l \varphi_{j}\left(v_{\rho}\right)-\operatorname{ord}_{\mathscr{F}_{l}}\left(v_{\rho}\right), \varphi_{j}\left(v_{\rho}\right)\right) & \text { if }(v, s)=\left(v_{\rho}, \varphi_{j}\left(v_{\rho}\right)\right), \\ \left(l \varphi_{j+1}\left(v_{\rho}\right)-\operatorname{ord}_{\mathscr{F}_{l}}\left(v_{\rho}\right), \varphi_{j+1}\left(v_{\rho}\right)\right) & \text { if }(v, s)=\left(v_{\rho}, \varphi_{j+1}\left(v_{\rho}\right)\right), \\ (d, 1) & \text { if }(v, s)=(0,1) .\end{cases}
$$

Definition 9.4. Suppose that $A(\underline{\varphi}, j, \theta) \neq \varnothing$. We denote by $B_{\underline{\varphi}, j, \theta}(\Lambda)$ the finite set

$$
\begin{aligned}
&\left\{\left(l \varphi_{j}\left(v_{\rho}\right)-\operatorname{ord}_{\mathscr{F}_{l}}\left(v_{\rho}\right), \varphi_{j}\left(v_{\rho}\right)\right) \mid \rho \leq \theta, \operatorname{dim} \rho=1\right\} \\
& \cup \begin{cases}\left\{\left(l \varphi_{j+1}\left(v_{\rho}\right)-\operatorname{ord}_{\mathscr{F}_{l}}\left(v_{\rho}\right), \varphi_{j+1}\left(v_{\rho}\right)\right) \mid \rho \in \Theta_{j+1}^{(1)}, \rho \subset \theta\right\} & \text { if } j \neq n, \\
\{(d, 1)\} & \text { if } j=n .\end{cases}
\end{aligned}
$$

Definition 9.5. If $A \subset \mathbb{Z}^{l+1}$ is a set, we denote by $F_{A}(x):=\sum_{a \in A} x^{a}$ the generating function of $A$; see [Cobo Pablos and González Pérez 2012, Section 12]. 
Proposition 9.6. We have the following equality:

$$
P_{\underline{\varphi}, j, \theta}(\Lambda)=(\mathbf{L}-1)^{l(j, \theta)} \sum_{a \in \mu(A(\underline{\varphi}, j, \theta))} \mathbf{L}^{\pi_{1}(a)} T^{\pi_{2}(a)} \in \mathbb{Z}[\mathbf{L}] \llbracket T \rrbracket .
$$

There exists a polynomial $R_{\underline{\varphi}, j, \theta} \in \mathbb{Z}[\mathbf{L}, T]$ such that $P_{\underline{\varphi}, j, \theta}(\Lambda)$ has the rational form

$$
P_{\underline{\varphi}, j, \theta}(\Lambda)=R_{\underline{\varphi}, j, \theta} \prod_{(a, b) \in B_{\underline{\varphi}, j, \theta}(\Lambda)}\left(1-\mathbf{L}^{a} T^{b}\right)^{-1} .
$$

Proof. The map $\mu$ defines a bijection

$$
A(\underline{\varphi}, j, \theta) / \sim \rightarrow \mu(A(\underline{\varphi}, j, \theta)), \quad[(v, s)] \mapsto \mu(v, s) ;
$$

see [Cobo Pablos and González Pérez 2012, Lemma 9.3] and Lemma 7.6. Then the equality (16) follows from the definitions.

We denote by $\pi_{*}: k \llbracket \mathbb{Z}^{l+1} \rrbracket \rightarrow k \llbracket \mathbf{L}, T \rrbracket$ the monomial transformation defined by $\pi_{*}\left(x^{a}\right):=\mathbf{L}^{\pi_{1}(a)} T^{\pi_{2}(a)}$ for $a \in \mathbb{Z}^{l+1}$. Then we get that

$$
P_{\underline{\varphi}, j, \theta}(\Lambda)=(\mathbf{L}-1)^{l(j, \theta)} \pi_{*}\left(F_{\mu\left(A_{\underline{\varphi}, j, \theta}\right)}(x)\right) .
$$

We apply [Cobo Pablos and González Pérez 2012, Theorem 12.4]. We obtain that the denominator of a rational form of $F_{\mu\left(A_{\varphi, j, \theta}\right)}(x)$ consists of products of terms $1-x^{\mu(b)}$ for $b$ running through the primitive integral vectors in the edges of the closure of the cone $\theta(\underline{\varphi}, j)$. Then the result follows by Remark 9.3 and Definition 9.4.

\section{Main results}

Definition 10.1. (i) For a semigroup $\Lambda$ generating a rank $d \geq 1$ lattice, we define a finite subset $B_{\mathrm{ar}}(\Lambda)$ of $\mathbb{Z}_{\geq 0}^{2}$ as (see Definition 9.4):

$$
B_{\mathrm{ar}}(\Lambda):=\bigcup_{\theta \in \bigcap_{r=1}^{j} \Theta_{r}, \theta \subset \mid \mathscr{\Phi}_{l(j, \theta) \mid}}^{1 \leq j \leq n} B_{\varphi, j, \theta}(\Lambda) .
$$

If $0<\eta<\sigma$, then $\Lambda \cap \eta^{\perp}$ is a semigroup generating lattice of rank $d-\operatorname{dim} \eta$. We use formula (17) and Definition 9.4 to define in this case a finite subset $B_{\text {ar }}\left(\Lambda \cap \eta^{\perp}\right)$ of $\mathbb{Z}_{\geq 0}^{2}$. We set

$$
B_{\mathrm{ar}}\left(\Lambda \cap \sigma^{\perp}\right):=\{(0,1)\} \quad \text { and } \quad B_{\mathrm{ar}, \Lambda}:=\bigcup_{\tau \leq \sigma} B_{\mathrm{ar}}\left(\Lambda \cap \sigma^{\perp}\right) .
$$

(ii) We define the integer

$$
q(\Lambda):=\operatorname{lcm}\left\{q(j, \theta)\left|\theta \in \bigcap_{r=1}^{j} \Theta_{r}, \theta \subseteq\right| D_{l(j, \theta)} \mid, \quad 1 \leq j \leq n\right\} .
$$


If $0<\eta<\sigma$, then $q\left(\Lambda \cap \eta^{\perp}\right)$ is the number obtained by replacing $\Lambda$ by $\Lambda \cap \eta^{\perp}$ in (18). We set $q\left(\Lambda \cap \sigma^{\perp}\right):=1$. We define also the integer

$$
q_{\Lambda}:=\operatorname{lcm}\left\{q\left(\Lambda \cap \eta^{\perp}\right) \mid \eta \leq \sigma\right\} .
$$

Theorem 10.2. Suppose that the field $k$ contains all $q(\Lambda)$-th roots of unity. Then there exists a polynomial $Q_{\mathrm{ar}}(\Lambda) \in \mathbb{Z}[\mathbf{L}, T]$ such that

$$
P_{\mathrm{ar}}(\Lambda)=\frac{1}{q(\Lambda)} Q_{\mathrm{ar}}(\Lambda) \prod_{(a, b) \in B_{\mathrm{ar}}(\Lambda)}\left(1-\mathbf{L}^{a} T^{b}\right)^{-1} .
$$

Proof. This follows from Propositions 8.5 and 9.6.

Notation 10.3. If $\eta<\sigma$, then the polynomial $Q_{\mathrm{ar}}\left(\Lambda \cap \eta^{\perp}\right)$ is obtained from Theorem 10.2 by replacing $\Lambda$ by the semigroup $\Lambda \cap \eta^{\perp}$. We set $Q_{\text {ar }}\left(\Lambda \cap \sigma^{\perp}\right):=1$.

Corollary 10.4. If the field $k$ contains all $q_{\Lambda}$-th roots of unity, then there exists a polynomial $Q_{\mathrm{ar}, \Lambda} \in \mathbb{Z}[\mathbf{L}, T]$ such that

$$
P_{\mathrm{ar}}^{\left(Z^{\Lambda}, 0\right)}(T)=\frac{1}{q_{\Lambda}} Q_{\mathrm{ar}, \Lambda} \prod_{(a, b) \in B_{\mathrm{ar}, \Lambda}}\left(1-\mathbf{L}^{a} T^{b}\right)^{-1} .
$$

Moreover, we have the equality

$$
P_{\mathrm{ar}}^{\left(Z^{\Lambda}, 0\right)}(T)=\sum_{\eta \leq \sigma} \frac{1}{q\left(\Lambda \cap \eta^{\perp}\right)} Q_{\mathrm{ar}}\left(\Lambda \cap \eta^{\perp}\right) \prod_{(a, b) \in B_{\mathrm{ar}}\left(\Lambda \cap \eta^{\perp}\right)}\left(1-\mathbf{L}^{a} T^{b}\right)^{-1} .
$$

Proof. The result follows by Theorem 10.2 and Proposition 8.1.

We can now compare the series $P_{\mathrm{geom}}^{(Z, 0)}(T)$ and $P_{\mathrm{ar}}^{(Z, 0)}(T)$ (see Definition 2.2). In [Cobo Pablos and González Pérez 2012] we introduced the series

$$
P_{\text {geom }}(\Lambda):=\sum_{s \geq 0} \chi_{c}\left(\left[j_{s}\left(H_{\Lambda}^{*}\right) \backslash \bigcup_{0 \neq \theta \leq \sigma} j_{s}\left(H_{\Lambda \cap \theta^{\perp}}\right)\right]\right) T^{s} \in K_{0}^{\text {mot }}\left(\operatorname{Var}_{k}\right) \otimes \mathbb{Q} \llbracket T \rrbracket,
$$

and we proved that

$$
P_{\text {geom }}^{\left(Z^{\Lambda}, 0\right)}(T)=\sum_{\theta \leq \sigma} P_{\text {geom }}\left(\Lambda \cap \theta^{\perp}\right) .
$$

Proposition 10.5. If the field $k$ contains all $q(\Lambda)$-th roots of unity, then

$$
P_{\text {ar }}(\Lambda)-P_{\text {geom }}(\Lambda)=\sum_{j=1}^{n} \sum_{\theta \in \bigcap_{r=1}^{j} \Theta_{r}}^{\theta \subset \mid \mathscr{D}_{l(j, \theta) \mid}}\left(1-\frac{1}{q(j, \theta)}\right) R_{\underline{\varphi}, j, \theta} \prod_{(a, b) \in B_{\underline{\varphi}, j, \theta}(\Lambda)}\left(1-\mathbf{L}^{a} T^{b}\right)^{-1} .
$$

Proof. This follows from Proposition 9.6, formula (20), Theorem 10.2, and the results in [Cobo Pablos and González Pérez 2012] for $P_{\text {geom }}(\Lambda)$. 
Corollary 10.6. If for every integer $1 \leq l \leq d$ and any vertex $v$ of the Newton polyhedra $\mathcal{N}\left(\mathscr{F}_{l}\right)$, there exists a subset $I_{v} \subset\{1, \ldots, n\}$ of l elements such that

$$
v=\sum_{i \in I_{v}} e_{i}
$$

and the vectors $e_{i}$ for $i \in I_{v}$ form part of a basis of $M$, then the series $P_{\mathrm{ar}}^{\left(Z^{\Lambda}, 0\right)}(T)$ and $P_{\mathrm{geom}}^{\left(Z^{\Lambda}, 0\right)}(T)$ coincide.

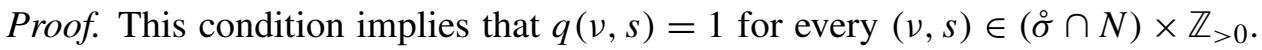
By Proposition 10.5, we get that $P_{\mathrm{ar}}(\Lambda)=P_{\text {geom }}(\Lambda)$. Now for any face $\eta \leq \sigma$, the vertices of the Newton polyhedra of the logarithmic jacobian ideals of $\Lambda \cap \eta^{\perp}$ are also vertices of the logarithmic jacobian ideals of $\Lambda$. The hypothesis implies that $\Lambda \cap \eta^{\perp}$ spans the lattice $M \cap \eta^{\perp}$ and then also that $P_{\text {ar }}\left(\Lambda \cap \eta^{\perp}\right)=P_{\text {geom }}\left(\Lambda \cap \eta^{\perp}\right)$.

Remark 10.7. Corollary 10.6 is a generalization of the Nicaise condition [2005b, Theorem 1] in the case of normal toric varieties.

Remark 10.8. The coordinates of the vectors in the set $B_{\varphi, j, \theta}(\Lambda)$ can be described geometrically in terms of the ideals $\mathscr{C}_{j}$ and $\mathscr{F}_{l}$ for $l=l(j, \theta)$. Let $\pi_{j}: Z_{j} \rightarrow Z^{\Lambda}$ be the composite of the normalization of $Z^{\Lambda}$, with the toric modification defined by the subdivision $\bigcap_{r=1}^{j} \Theta_{r}$ of $\sigma$. The modification $\pi_{j}$ is the minimal toric modification that factors through the normalized blowing up with center $\mathscr{C}_{r}$ for $r=1, \ldots, j$. If $\rho$ is an edge of $\theta$, the orbit closure $E_{\rho}$ of the orbit associated to $\rho$ on $Z_{j}$ has codimension one. We denote by $v_{\rho}$ the divisorial valuation defined by $E_{\rho}$. It satisfies $v_{\rho}\left(X^{m}\right)=\left\langle v_{\rho}, m\right\rangle$ for $m \in M$. The pullback $\pi_{j}^{*}(\mathscr{I})$ of a monomial ideal $\mathscr{I}$ of $Z^{\Lambda}$ is a sheaf of monomial ideals on $Z_{j}$. The ideals $\pi_{j}^{*}\left(\mathscr{C}_{r}\right)$ for $r=1, \ldots, j$ are locally principal on $Z_{j}$. Then we get the following identities:

$$
\begin{aligned}
\varphi_{j}\left(v_{\rho}\right) & =v_{\rho}\left(\pi_{j}^{*}\left(\mathscr{C}_{j}\right)\right)-v_{\rho}\left(\pi_{j}^{*}\left(\mathscr{C}_{j-1}\right)\right), \\
\varphi_{j+1}\left(v_{\rho}\right) & =v_{\rho}\left(\pi_{j}^{*}\left(\mathscr{C}_{j+1}\right)\right)-v_{\rho}\left(\pi_{j}^{*}\left(\mathscr{C}_{j}\right)\right), \\
\operatorname{ord}_{\mathscr{F}_{l}}\left(v_{\rho}\right) & =v_{\rho}\left(\pi_{j}^{*}\left(\mathscr{F}_{l}\right)\right) .
\end{aligned}
$$

Compare this with the geometrical description of the set of candidate poles of $P_{\text {geom }}^{\left(Z^{\Lambda}, 0\right)}(T)$; see [Cobo Pablos and González Pérez 2012].

\section{The normal case}

In the normal case, when the semigroup $\Lambda$ is saturated, that is, $\Lambda=\sigma^{\vee} \cap M$, we describe the motivic arithmetic series in a simpler way by using $j^{s}\left(H_{\Lambda}\right)=j_{s}\left(H_{\Lambda}^{*}\right)$; see [Nicaise 2005b].

Notation 11.1. (i) $\mathscr{A}=\bigsqcup_{l=1}^{d} \bigsqcup_{\tau \in \bigcap_{r=1}^{l} \Sigma_{r}} A(\underline{\phi}, l, \tau) / \sim$.

(ii) For $s_{0} \geq 0$ we set $\mathscr{A}_{s_{0}}=\left\{[(\nu, s)] \in \mathscr{A} \mid s=s_{0}\right\}$. 
Remark 11.2. The set $\mathscr{A}_{s}$ is finite; see [Cobo Pablos and González Pérez 2012, Remark 8.2]. By (9) and Lemma 7.6, we deduce that

$$
\mathscr{A}=\bigsqcup_{j=1}^{n} \bigsqcup_{\theta \in \bigcap_{r=1}^{j} \Theta_{r}} A(\underline{\varphi}, j, \theta) / \sim
$$

Proposition 11.3. Let us fix an integer $s_{0} \geq 1$. The set $j_{s_{0}}\left(H^{*}\right)$ expresses as a finite disjoint union of locally closed subsets as $j_{s_{0}}\left(H^{*}\right)=\bigsqcup_{[(v, s)] \in A_{s_{0}}} j_{s_{0}}\left(H_{v}^{*}\right)$. We deduce that $\chi_{f}\left(\left[j_{s}\left(H^{*}\right)\right]_{f}\right)=\sum_{[(v, s)] \in \mathscr{A}_{s}} \chi_{f}\left(\left[j_{s}\left(H_{v}^{*}\right)\right]_{f}\right)$.

Proof. The first claim follows by the method of [Cobo Pablos and González Pérez 2012, Proposition 8.11]. The second is a consequence of the first and Proposition 5.2.

Theorem 11.4. If $Z^{\Lambda}$ is normal, then we have

$$
P_{\mathrm{ar}}^{\left(Z^{\Lambda}, 0\right)}=\sum_{j=1}^{n} \sum_{\theta \in \bigcap_{r=1}^{j} \Theta_{r}} \frac{1}{q(j, \theta)} R_{\underline{\varphi}, j, \theta} \prod_{(a, b) \in B_{\underline{\varphi}, j, \theta}(\Lambda)}\left(1-\mathbf{L}^{a} T^{b}\right)^{-1} .
$$

Proof. It is a consequence of Propositions 11.3 and 9.6 and Remark 11.2.

Corollary 11.5. Suppose that the affine toric variety $Z^{\Lambda}$ is normal. If $\theta \leq \sigma$, we denote by $\sigma_{\theta}^{\vee}$ the image of the cone $\sigma^{\vee}$ in $\left(M_{\theta}\right)_{\mathbb{R}}$, where $M_{\theta}:=M / \theta^{\perp} \cap M$, and by $\Lambda(\theta)$ the saturated semigroup $\Lambda(\theta):=\left(\sigma_{\theta}^{\vee} \cap M_{\theta}\right) \times \mathbb{Z}_{\geq 0}^{\operatorname{codim} \theta}$. With this notation, we have

$$
P_{\mathrm{ar}}^{Z^{\Lambda}}(T)=\sum_{\theta \leq \sigma}(\mathbf{L}-1)^{\operatorname{codim} \theta} P_{\mathrm{ar}}^{\left(Z^{\Lambda(\theta)}, 0\right)}(T) .
$$

Proof. The proof follows by the arguments of [Cobo Pablos and González Pérez 2012, Corollary 4.11].

\section{Examples}

12a. The case of monomial curves. Let $\Lambda \subset \mathbb{Z}_{\geq 0}$ be a semigroup with minimal system of generators $e_{1}<e_{2}<\cdots<e_{n}$ such that $\operatorname{gcd}\left\{e_{1}, \ldots, e_{n}\right\}=1$. In this case, we have $\stackrel{\circ}{\circ} \cap N=\mathbb{Z}_{>0}$. If $q_{i}:=\operatorname{gcd}\left\{e_{1}, \ldots, e_{i}\right\}$, then we obtain

$$
P_{\mathrm{ar}}^{\left(Z^{\Lambda}, 0\right)}(T)=\frac{1}{1-T}+\frac{\mathbf{L}-1}{1-\mathbf{L} T}\left(\frac{1}{q_{1}} \frac{T^{e_{1}}}{1-T^{e_{1}}}+\sum_{i=2}^{n} \frac{q_{i-1}-q_{i}}{q_{i-1} q_{i}} \frac{\mathbf{L}^{e_{i}-e_{1}} T^{e_{i}}}{1-\mathbf{L}^{e_{i}-e_{1}} T^{e_{i}}}\right) .
$$

This follows from the results of this paper, taking the following observations into account:

- We have the equality $j_{s}(H)=j_{s}\left(H^{*}\right)$.

- If $v, v^{\prime} \in \mathbb{Z}_{>0}$ satisfy $j_{s}\left(H_{v}^{*}\right)$ and $j_{s}\left(H_{v^{\prime}}^{*}\right) \neq\{0\}$, then the equality $j_{s}\left(H_{v}^{*}\right)=$ $j_{s}\left(H_{v^{\prime}}^{*}\right)$ implies $v=v^{\prime}$. 
- If $v \in \mathbb{Z}_{>0}$ satisfies $v e_{i} \leq s<v e_{i+1}$, then $q(v, s)=q_{i}$.

Then, setting $e_{d+1}:=\infty$, we get the following equality, which implies (21):

$$
P_{\mathrm{ar}}^{\left(Z^{\Lambda}, 0\right)}(T)=\frac{1}{1-T}+\sum_{i=1}^{n} \sum_{\nu=1}^{\infty} \sum_{s=\nu e_{i}}^{\nu e_{i+1}-1}(\mathbf{L}-1) \frac{1}{q_{i}} \mathbf{L}^{s-v e_{1}} T^{s} .
$$

Remark 12.1. The inequalities $q_{1} \geq q_{2} \geq \cdots \geq q_{n}=1$ are not always strict. For instance, if $\Lambda$ is generated by $e_{1}=8, e_{2}=18, e_{3}=20$ and $e_{4}=21$, then we get $q_{1}=8, q_{2}=q_{3}=2$ and $q_{4}=1$. It follows from (21) that the term $1-\mathbf{L}^{12} T^{20}$ is not a factor of the denominator of the series $P_{\mathrm{ar}}^{\left(Z^{\Lambda}, 0\right)}(T)$. If $\Lambda^{\prime}$ is the semigroup generated by $e_{1}, e_{2}$ and $e_{4}$, then we obtain from (21) that

$$
P_{\mathrm{ar}}^{\left(Z^{\Lambda}, 0\right)}(T)=P_{\mathrm{ar}}^{\left(Z^{\Lambda^{\prime}}, 0\right)}(T),
$$

while the semigroups $\Lambda$ and $\Lambda^{\prime}$ are not isomorphic. In contrast with this behavior, the motivic series $P_{\mathrm{ar}}^{(C, 0)}(T)$ of a plane branch $(C, 0)$ determines the semigroup of the branch $(C, 0)$; see [DL 2001].

12b. An example of non-normal toric surface. Consider the semigroup $\Lambda$ generated by the vectors $e_{1}=(5,0), e_{2}=(0,2), e_{3}=(0,3)$ and $e_{4}=(6,2)$. The cone $\sigma$ is $\mathbb{R}_{\geq 0}^{2}$ and the lattice $M$ is equal to $\mathbb{Z}^{2}$. We have the semigroups $\Lambda \cap \eta_{1}^{\perp}=(5,0) \mathbb{Z}_{>0}$ and $\Lambda \cap \eta_{2}^{\perp}=(0,2) \mathbb{Z}_{>0}+(0,3) \mathbb{Z}_{>0}$, where $\eta_{1}$ and $\eta_{2}$ are the one-dimensional faces of $\sigma$. By the case of monomial curves, we get

$$
P_{\mathrm{ar}}\left(\Lambda \cap \eta_{1}^{\perp}\right)=\frac{\mathbf{L}-1}{1-\mathbf{L} T} \frac{T}{1-T} \quad \text { and } \quad P_{\mathrm{ar}}\left(\Lambda \cap \eta_{2}^{\perp}\right)=\frac{\mathbf{L}-1}{2(1-\mathbf{L} T)}\left(\frac{T^{2}}{1-T^{2}}+\frac{\mathbf{L} T^{3}}{1-\mathbf{L} T^{3}}\right) .
$$

Figure 1 shows the subdivisions associated with the ideals $\mathscr{C}_{r}$ for $r=1,2,3$.

In the following table, we give the different values of $q(j, \theta)$ and $l(j, \theta)$ for $\theta$ in the subdivisions of Figure 1 and $j$ such that $A(\underline{\varphi}, j, \theta) \neq \varnothing$. We exclude from

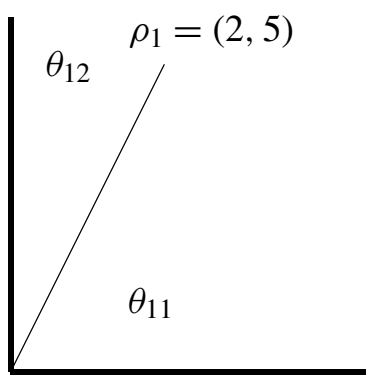

$\Theta_{1}$

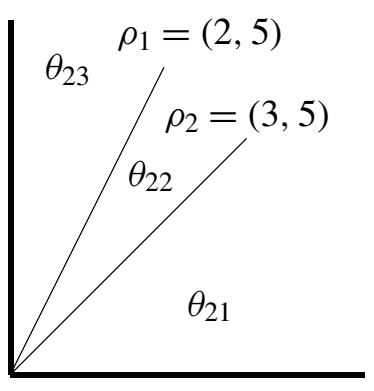

$\Theta_{1} \cap \Theta_{2}$

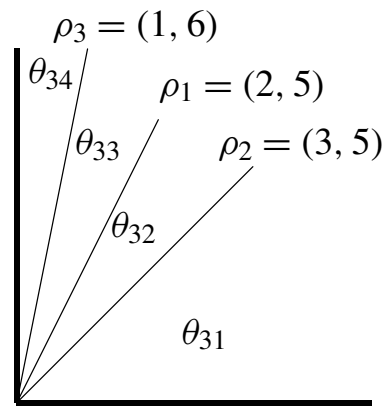

$\Theta_{1} \cap \Theta_{2} \cap \Theta_{3}$

Figure 1. The subdivisions $\Theta_{1}, \Theta_{1} \cap \Theta_{2}$ and $\Theta_{1} \cap \Theta_{2} \cap \Theta_{3}$. 
this table the cones in $\theta \in \bigcap_{r=1}^{4} \Theta_{r}$ for $j=4$, since in this case $q(4, \theta)=1$ and $l(4, \theta)=2$.

\begin{tabular}{lllll}
$q\left(1, \theta_{11}\right)=2$ & $q\left(1, \theta_{12}\right)=5$ & & & \\
$l\left(1, \theta_{11}\right)=1$ & $l\left(1, \theta_{12}\right)=1$ & & \\
\hline$q\left(2, \theta_{21}\right)=1$ & $q\left(2, \theta_{22}\right)=10$ & $q\left(2, \theta_{23}\right)=10$ & $q\left(2, \rho_{1}\right)=10$ & \\
$l\left(2, \theta_{21}\right)=1$ & $l\left(2, \theta_{22}\right)=2$ & $l\left(2, \theta_{23}\right)=2$ & $l\left(2, \rho_{1}\right)=2$ & \\
\hline$q\left(3, \theta_{31}\right)=5$ & $q\left(3, \theta_{32}\right)=5$ & $q\left(3, \theta_{33}\right)=5$ & $q\left(3, \theta_{34}\right)=2$ & $q\left(3, \rho_{1}\right)=5$ \\
& & & & $q\left(3, \rho_{2}\right)=5$ \\
$l\left(3, \theta_{31}\right)=2$ & $l\left(3, \theta_{32}\right)=5$ & $l\left(3, \theta_{33}\right)=2$ & $l\left(3, \theta_{34}\right)=2$ & $l\left(3, \rho_{1}\right)=2$ \\
& & & & $l\left(3, \rho_{2}\right)=2$ \\
\hline
\end{tabular}

Notice that $A\left(\varphi, 1, \rho_{1}\right)=A\left(\varphi, 2, \rho_{2}\right)=A\left(\varphi, 3, \rho_{3}\right)=\varnothing$. In the following table, we have filled in the cases corresponding to the pairs $(a, b) \in B_{\text {ar }}(\Lambda),(a, b) \neq(2,1)$ in terms of the rays appearing in the subdivisions of Figure 1:

\begin{tabular}{lccccc}
$(a, b) \in B_{\mathrm{ar}}(\Lambda)$ & $v_{\rho_{1}}=(2,5)$ & $v_{\rho_{2}}=(3,5)$ & $v_{\rho_{3}}=(1,6)$ & $v_{\sigma^{\vee} \cap \eta_{1}^{\perp}}=(1,0)$ & $v_{\sigma^{\vee} \cap \eta_{2}^{\perp}}=(0,1)$ \\
\hline$\left(2 \varphi_{2}-\operatorname{ord}_{\mathscr{q}_{2}}, \varphi_{2}\right)$ & $(0,10)$ & $(5,15)$ & & & $(2,2)$ \\
$\left(2 \varphi_{3}-\operatorname{ord}_{q_{2}}, \varphi_{3}\right)$ & $(10,15)$ & $(5,15)$ & $(19,18)$ & $(5,5)$ & $(2,2)$ \\
$\left(2 \varphi_{4}-\operatorname{ord}_{q_{2}}, \varphi_{4}\right)$ & $(24,22)$ & $(31,28)$ & $(19,18)$ & $(7,6)$ & $(4,3)$ \\
\hline
\end{tabular}

It follows that $B_{\mathrm{ar}, \Lambda}=B_{\mathrm{ar}}(\Lambda) \cup\{(1,3),(0,2),(1,1),(0,1)\}$. We have computed the sum of the series $P_{\mathrm{ar}}^{\left(Z^{\Lambda}, 0\right)}(T)$ with the methods of [Cobo Pablos and González Pérez 2012]. We have obtained an irredundant representation of the form

$$
P_{\mathrm{ar}}^{\left(Z^{\Lambda}, 0\right)}(T)=R(\mathbf{L}, T) \prod_{(a, b) \in B}\left(1-\mathbf{L}^{a} T^{b}\right)^{-1}
$$

with $R(\mathbf{L}, T) \in \mathbb{Q}[\mathbf{L}, T]$, and where $B=B_{\mathrm{ar}, \Lambda} \backslash\{(24,22),(31,28)\}$.

\section{References}

[Bittner 2004] F. Bittner, "The universal Euler characteristic for varieties of characteristic zero", Compos. Math. 140:4 (2004), 1011-1032. MR 2005d:14031 Zbl 1086.14016

[Cobo Pablos and González Pérez 2012] H. Cobo Pablos and P. D. González Pérez, "Motivic Poincaré series, toric singularities and logarithmic Jacobian ideals", J. Algebraic Geom. 21:3 (2012), 495-529. MR 2914802 Zbl 06051369

[CoGP 2010] H. Cobo Pablos and P. D. González Pérez, "Geometric motivic Poincaré series of quasiordinary sigularities”, Math. Proc. Cambridge Philos. Soc. 149:1 (2010), 49-74. MR 2011j:14032 Zbl 1198.14015

[Denef 1984] J. Denef, "The rationality of the Poincaré series associated to the $p$-adic points on a variety”, Invent. Math. 77:1 (1984), 1-23. MR 86c:11043 Zbl 0537.12011 
[DL 1999] J. Denef and F. Loeser, "Germs of arcs on singular algebraic varieties and motivic integration”, Invent. Math. 135:1 (1999), 201-232. MR 99k:14002 Zbl 0928.14004

[DL 2001] J. Denef and F. Loeser, "Definable sets, motives and p-adic integrals", J. Amer. Math. Soc. 14:2 (2001), 429-469. MR 2002k:14033 Zbl 1040.14010

[DL 2002] J. Denef and F. Loeser, "Motivic integration and the Grothendieck group of pseudo-finite fields", pp. 13-23 in Proceedings of the International Congress of Mathematicians (Beijing, 2002), vol. 2, edited by T. Li, Higher Education Press, Beijing, 2002. MR 2004f:14040 Zbl 1101.14029

[DL 2004] J. Denef and F. Loeser, "On some rational generating series occurring in arithmetic geometry”, pp. 509-526 in Geometric aspects of Dwork theory (Padova, 2001), vol. 1, edited by A. Adolphson et al., Walter de Gruyter, Berlin, 2004. MR 2005h:11267 Zbl 1061.11067

[Ewald 1996] G. Ewald, Combinatorial convexity and algebraic geometry, Graduate Texts in Mathematics 168, Springer, New York, 1996. MR 97i:52012 Zbl 0869.52001

[Fulton 1993] W. Fulton, Introduction to toric varieties, Annals of Mathematics Studies 131, Princeton University Press, 1993. MR 94g:14028 Zbl 0813.14039

[Gel'fand et al. 1994] I. M. Gel'fand, M. M. Kapranov, and A. V. Zelevinsky, Discriminants, resultants, and multidimensional determinants, Birkhäuser, Boston, MA, 1994. MR 95e:14045 Zbl 0827.14036

[Gillet and Soulé 1996] H. Gillet and C. Soulé, "Descent, motives and K-theory", J. Reine Angew. Math. 478 (1996), 127-176. MR 98d:14012 Zbl 0863.19002

[Greenberg 1966] M. J. Greenberg, "Rational points in Henselian discrete valuation rings", Inst. Hautes Études Sci. Publ. Math. 31 (1966), 59-64. MR 34 \#7515 Zbl 0146.42201

[Guillén and Navarro Aznar 2002] F. Guillén and V. Navarro Aznar, "Un critère d'extension des foncteurs définis sur les schémas lisses", Publ. Math. Inst. Hautes Études Sci. 95 (2002), 1-91. MR 2004i:14020 Zbl 1075.14012

[Ishii 2004] S. Ishii, "The arc space of a toric variety", J. Algebra 278:2 (2004), 666-683. MR 2005f: 14100 Zbl 1073.14066

[Ishii 2005] S. Ishii, “Arcs, valuations and the Nash map”, J. Reine Angew. Math. 588 (2005), 71-92. MR 2006k:14005 Zbl 1082.14007

[Lejeune-Jalabert and Reguera 2003] M. Lejeune-Jalabert and A. J. Reguera, “The Denef-Loeser series for toric surface singularities", Rev. Mat. Iberoamericana 19:2 (2003), 581-612. MR 2004m: 14001 Zbl 1058.14006

[Nicaise 2005a] J. Nicaise, "Arcs and resolution of singularities", Manuscripta Math. 116:3 (2005), 297-322. MR 2006h:14019 Zbl 1073.14050

[Nicaise 2005b] J. Nicaise, "Motivic generating series for toric surface singularities", Math. Proc. Cambridge Philos. Soc. 138:3 (2005), 383-400. MR 2006d:14012 Zbl 1075.14020

[Oda 1988] T. Oda, Convex bodies and algebraic geometry: An introduction to the theory of toric varieties, Ergebnisse der Mathematik und ihrer Grenzgebiete (3) 15, Springer, Berlin, 1988. MR 88m:14038 Zbl 0628.52002

[Rond 2009] G. Rond, "Séries de Poincaré motiviques d'un germe d'hypersurface irréductible quasiordinaire", pp. 371-396 in Présentation [Équations différentielles et singularités: En l'honneur de J. M. Aroca], edited by F. Cano et al., Astérisque 323, Société Mathématique de France, Paris, 2009. MR 2011c:14039 Zbl 1190.14008

[Scholl 1994] A. J. Scholl, "Classical motives", pp. 163-187 in Motives (Seattle, WA, 1991), edited by U. Jannsen et al., Proc. Sympos. Pure Math. 55, American Mathematical Society, Providence, RI, 1994. MR 95b:11060 Zbl 0814.14001 
Communicated by Joseph Gubeladze

Received 2011-11-07 Revised 2012-01-31 Accepted 2012-03-03

helena.cobo@wis.kuleuven.ac.be Department of Mathematics, University of Leuven, Celestijnenlaan 200B, B-3001 Heverlee, Belgium

pgonzalez@mat.ucm.es Instituto de Ciencias Matemáticas, Departamento de Álgebra, Facultad de Ciencias Matemáticas, Universidad Complutense de Madrid, Plaza de las Ciencias 3, 28040 Madrid, Spain 


\section{Algebra \& Number Theory}

msp.org/ant

\section{EDITORS}

MANAGING EDITOR

Bjorn Poonen

Massachusetts Institute of Technology

Cambridge, USA

\author{
EDITORIAL BOARD CHAIR \\ David Eisenbud \\ University of California \\ Berkeley, USA
}

\section{BOARD OF EDITORS}

Georgia Benkart

Dave Benson

Richard E. Borcherds

John H. Coates

J-L. Colliot-Thélène

Brian D. Conrad

Hélène Esnault

Hubert Flenner

Edward Frenkel

Andrew Granville

Joseph Gubeladze

Ehud Hrushovski

Craig Huneke

Mikhail Kapranov

Yujiro Kawamata

János Kollár

Yuri Manin

Barry Mazur

Philippe Michel
University of Wisconsin, Madison, USA

University of Aberdeen, Scotland

University of California, Berkeley, USA

University of Cambridge, UK

CNRS, Université Paris-Sud, France

University of Michigan, USA

Freie Universität Berlin, Germany

Ruhr-Universität, Germany

University of California, Berkeley, USA

Université de Montréal, Canada

San Francisco State University, USA

Hebrew University, Israel

University of Virginia, USA

Yale University, USA

University of Tokyo, Japan

Princeton University, USA

Northwestern University, USA

Harvard University, USA

École Polytechnique Fédérale de Lausanne
Susan Montgomery

Shigefumi Mori

Raman Parimala

Jonathan Pila

Victor Reiner

Karl Rubin

Peter Sarnak

Joseph H. Silverman

Michael Singer

Vasudevan Srinivas

J. Toby Stafford

Bernd Sturmfels

Richard Taylor

Ravi Vakil

Michel van den Bergh

Marie-France Vignéras

Kei-Ichi Watanabe

Efim Zelmanov
University of Southern California, USA

RIMS, Kyoto University, Japan

Emory University, USA

University of Oxford, UK

University of Minnesota, USA

University of California, Irvine, USA

Princeton University, USA

Brown University, USA

North Carolina State University, USA

Tata Inst. of Fund. Research, India

University of Michigan, USA

University of California, Berkeley, USA

Harvard University, USA

Stanford University, USA

Hasselt University, Belgium

Université Paris VII, France

Nihon University, Japan

University of California, San Diego, USA

\section{PRODUCTION}

production@msp.org

Silvio Levy, Scientific Editor

See inside back cover or msp.org/ant for submission instructions.

The subscription price for 2013 is US \$200/year for the electronic version, and \$350/year ( $\$ 40$, if shipping outside the US) for print and electronic. Subscriptions, requests for back issues and changes of subscribers address should be sent to MSP.

Algebra \& Number Theory (ISSN 1944-7833 electronic, 1937-0652 printed) at Mathematical Sciences Publishers, 798 Evans Hall \#3840, c/o University of California, Berkeley, CA 94720-3840 is published continuously online. Periodical rate postage paid at Berkeley, CA 94704, and additional mailing offices.

ANT peer review and production are managed by EditFLOW ${ }^{\circledR}$ from Mathematical Sciences Publishers.

\section{PUBLISHED BY}

mathematical sciences publishers

nonprofit scientific publishing

http://msp.org/

(C) 2013 Mathematical Sciences Publishers 


\section{Algebra \& Number Theory}

$\begin{array}{lll}\text { Volume } 7 & \text { No. } 2 & 2013\end{array}$

The system of representations of the Weil-Deligne group associated to an abelian variety 243 RUTGER NOOT

Fourier-Jacobi coefficients of Eisenstein series on unitary groups

BEI ZHANG

The phase limit set of a variety

MoUnIR NisSE and FRANK SoTTILE

Base change behavior of the relative canonical sheaf related to higher dimensional moduli

Zsolt PATAKFALVI

Two ways to degenerate the Jacobian are the same

JESSE LEO KASS

Arithmetic motivic Poincaré series of toric varieties

Helena Cobo Pablos and Pedro Daniel González Pérez

Maximal ideals and representations of twisted forms of algebras

Michael LAU and ARTURo PiAnZOLA

Higher Chow groups of varieties with group action 